\title{
The flag polynomial of the Minkowski sum of simplices
}

\author{
Geir Agnarsson *
}

\begin{abstract}
For a polytope we define the flag polynomial, a polynomial in commuting variables related to the well-known flag vector and describe how to express the the flag polynomial of the Minkowski sum of $k$ standard simplices in a direct and canonical way in terms of the $k$-th master polytope $P(k)$ where $k \in \mathbb{N}$. The flag polynomial facilitates many direct computations. To demonstrate this we provide two examples; we first derive a formula for the $f$-polynomial and the maximum number of $d$-dimensional faces of the Minkowski sum of two simplices. We then compute the maximum discrepancy between the number of $(0, d)$-chains of faces of a Minkowski sum of two simplices and the number of such chains of faces of a simple polytope of the same dimension and on the same number of vertices.
\end{abstract}

2010 MSC: 05A15, 05A16, 52B05, 52B11.

Keywords: polytope, Minkowski sum, master polytope, flag polynomial, $f$-polynomial.

\section{Introduction and motivation}

For a polytope $P$ of dimension $d$, the face vector of $P$ is given by $f(P)=\left(f_{0}(P), \ldots, f_{d-1}(P)\right)$ where $f_{i}(P)$ is the number of $i$-dimensional faces of $P$. It is an ongoing investigation to describe the face vectors for $d$-dimensional convex polytopes. The case $d \leq 3$ are the only cases where this description is complete. A nice theorem by Steinitz [1] states that an integer vector $\left(f_{0}, f_{1}, f_{2}\right)$ is a face vector for a three dimensional polytope $P$ if and only if (i) we have the Euler relation $f_{0}-f_{1}+f_{2}=2$, (ii) $f_{2}-4 \leq 2\left(f_{0}-4\right)$ and (iii) $f_{0}-4 \leq 2\left(f_{2}-4\right)$. Moreover, the first inequality is tight if and only if $P$ is simplicial, the second one is tight if and only if $P$ is simple. The only linear equation satisfied by the entries of the face vector is the Euler-Poincare relation $\sum_{i=0}^{d-1}(-1)^{i} f_{i}=1+(-1)^{d-1}$, that holds for all polytopes of dimension $d$. For higher dimensions $d \geq 4$ no such nice description of face vectors has been found yet. In the hope of obtaining a description of a polytope in terms of parameters/entries of a vector, there is the flag vector $\left(f_{S}(P)\right)_{S \subseteq\{0,1, \ldots, d-1\}}$, containing $2^{d}$ entries where for each $S=\left\{s_{1}, s_{2}, \ldots, s_{\ell}\right\} \subseteq\{0, \ldots, d-1\}$ the entry $f_{S}(P)$ denotes the number of strict chains (or flags) $A_{1} \subset A_{2} \subset \cdots \subset A_{\ell}$ of faces of $P$ where $\operatorname{dim}\left(A_{i}\right)=s_{i}$. The entries $f_{S}(P)$ do satisfy the generalized Dehn-Sommerville equations which, together with the existence of matching polytopes, implies that the linear span of flag vectors of $d$-dimensional polytopes is precisely $F_{d}$, the $d$-th Fibonacci number, as shown in the seminal paper [2]. One way to encode the information contained in the flag vector of a $d$-polytope $P$ is to define the ab-index, a linear combination of non-commuting monomials in $\mathbf{a}$ and $\mathbf{b}$ of degree $d$ in the following way: for $S \subseteq\{0,1, \ldots, d-1\}$ let $w_{S}=t_{0} t_{1} \cdots t_{d-1}$, where $t_{i}=\mathbf{a}$ if $i \notin S$ and

* Department of Mathematical Sciences, George Mason University, MS 3F2, 4400 University Drive, Fairfax, VA 22030, USA, geir@math.gmu.edu 
$t_{i}=\mathbf{b}$ if $i \in S$. The ab-index $\Psi(P)$ for $P$ is then defined as $\Psi(P)=\sum_{S} h_{S}(P) w_{S}$, where the sum is over all $S \subseteq\{0,1, \ldots, d-1\}$ and $h_{S}(P)=\sum_{T \subseteq S}(-1)^{|S \backslash T|} f_{T}(P)$. An elegant consequence of the generalized Dehn-Sommerville equations for the entries $f_{S}(P)$ is that each ab-index can be written as a non-commuting polynomial in $\mathbf{c}=\mathbf{a}+\mathbf{b}$ and $\mathbf{d}=\mathbf{a b}+\mathbf{b a}$. The resulting polynomial called the cd-index, also denoted $\Psi(P)$ and first appeared in 3 . Within the free non-commuting $\mathbb{R}$-algebra $\mathbb{R}\langle\mathbf{a}, \mathbf{b}\rangle$ in two variables $\mathbf{a}$ and $\mathbf{b}$, the degree of $\mathbf{c}$ is set to be 1 and the degree of $\mathbf{d}$ is set to be two. In this way the number of all homogeneous monomials of degree $d$ in $\mathbf{c}$ and $\mathbf{d}$ is precisely $F_{d}$. Hence, the cd-index is the most compact way to encode all the entries of the flag vector of a polytope $P$ w.r.t. linear relations of the coefficients of the monomial in $\mathbf{c}$ and $\mathbf{d}$. A lot of current research is therefore directed toward understanding the cd-index for polytopes. Although the cd-index is an elegant and compact way to encode the flag vector for a polytope $P$, it can be at times difficult to handle in many explicit computations since algebraic manipulation in $\mathbb{R}\langle\mathbf{c}, \mathbf{d}\rangle \subseteq \mathbb{R}\langle\mathbf{a}, \mathbf{b}\rangle$ can become particularly cumbersome.

The purpose of this article is to do the opposite of the above: namely, instead of attempting to compactify the encoding of the flag vector further, then we shall instead relax the constrictions and allow some redundancies among coefficients in order to facilitate direct computations. This will in certain cases allow us to write down explicit (albeit involved) formulae from which the entries of the flag vector can be obtained. We will define the flag polynomial for a polytope, a polynomial in commuting variables that will behave reasonably well when constructing polytopes by known methods i.e. prisms, pyramids, products, free joins and Minkowski sums.

The article is organized as follows: In Section 2 we present our basic definitions and write some direct consequences. In Section 3 we state (but do not prove) Theorem 3.3 , the main result of the section; a formula for the flag polynomial of a Minkowski sum of $k$ standard simplices. We then parse through an explicit application of Theorem 3.3 and note some consequences. Section 4 is devoted to the derivation of Theorem 4.7, a formula for the flag polynomial of a polytope $P\left(Q_{1}, \ldots, Q_{m}\right)$, a polytope obtained when the geometric polytope $P$ in $\mathbb{R}^{m}$ acts on the polytopes $Q_{1}, \ldots, Q_{m}$. This action generalizes the notion of the free join of two polytopes. Finally, we note that Theorem 3.3 is a corollary of Theorem 4.7. In the last Section [5 we present two examples on the application of the flag polynomial. In the first example we compute the maximum number of faces of an arbitrary Minkowski sum of two simplices. In the latter example we compute the maximum discrepancy between the number of $(0, d)$-chains of faces of a Minkowski sum of two simplices and the number of $(0, d)$-chains of faces of a simple polytope of the same dimension and on the same number of vertices as the Minkowski sum.

This article can be viewed as a self contained continuation of [8]. For related references and history of the significance of Minkowski sums of simplices we refer to the introduction of [8]. Also, for self-containment we will present Definition 3.1 and some terminology from [8].

For $r \in \mathbb{N}$ we let $[r]=\{1,2, \ldots, r\}$. The standard simplex $\Delta_{r-1}=\Delta_{[r]}$ of dimension $r-1$ is given by $\Delta_{r-1}=\Delta_{[r]}=\left\{\tilde{x}=\left(x_{1}, \ldots, x_{r}\right) \in \mathbb{R}^{r}: x_{i} \geq 0\right.$ for all $\left.i, x_{1}+\cdots+x_{r}=1\right\}$. Each subset $F \subseteq[r]$ yields a face $\Delta_{F}$ of $\Delta_{[r]}$ given by $\Delta_{F}=\left\{\tilde{x} \in \Delta_{[r]}: x_{i}=0\right.$ for $\left.i \notin F\right\}$. Clearly $\Delta_{F}$ is itself a simplex embedded in $\mathbb{R}^{r}$. If $\mathcal{F}$ is a family of subsets of $[r]$, then we can form the Minkowski sum of simplices

$$
P_{\mathcal{F}}=\sum_{F \in \mathcal{F}} \Delta_{F}=\left\{\sum_{F \in \mathcal{F}} \tilde{x}_{F}: \tilde{x}_{F} \in \Delta_{F} \text { for each } F \in \mathcal{F}\right\} .
$$

Note that a face of any polytope $P \subseteq \mathbb{R}^{r}$ (in particular of $\Delta_{[r]}$ ) is given by the set of points that maximize a linear functional $c^{T} \tilde{x}$ for $\tilde{x} \in P$. 
Recall the $f$-polynomial of a polytope: let $P$ be a $d$-dimensional polytope. For each $i \in$ $\{0,1, \ldots, d\}$ let $\mathbf{f}_{i}(P)$ denote the set of all $i$-dimensional faces of $P$ and so $f_{i}(P)=\left|\mathbf{f}_{i}(P)\right|$ is the number of $i$-dimensional faces of $P$. Then $\mathbf{f}(P)=\bigcup_{i=0}^{d} \mathbf{f}_{i}(P)$ is the set of all the faces of $P$ except the empty face. The $f$-polynomial of $P$ is then given by $f_{P}(x)=\sum_{i=0}^{d} f_{i}(P) x^{i}$.

Observation 1.1 The $f$-polynomial for the standard $(r-1)$-dimensional simplex $\Delta_{r-1}=\Delta_{[r]}$ is given by

$$
f_{\Delta_{r-1}}(x)=\frac{(x+1)^{r}-1}{x} .
$$

\section{Definitions and some basic properties}

We now define our main object for this paper, a generalization of the $f$-polynomial of a polytope, and derive some basic results.

Conventions: (i) For an $\ell$-tuple $\tilde{x}=\left(x_{1}, \ldots, x_{\ell}\right)$ of variables and an $\ell$-tuple of numbers $\tilde{a}=$ $\left(a_{1}, \ldots, a_{\ell}\right)$, let $\tilde{x}^{\tilde{a}}=x_{1}^{a_{1}} x_{2}^{a_{2}} \cdots x_{\ell}^{a_{\ell}}$. (ii) For $\tilde{a}=\left(a_{1}, \ldots, a_{\ell}\right)$ let $\partial(\tilde{a})=\left(a_{1}, a_{2}-a_{1}, a_{3}-a_{2}, \ldots, a_{\ell}-\right.$ $\left.a_{\ell-1}\right)$.

Definition 2.1 Let $P$ be a polytope with $\operatorname{dim}(P)=d$ and $\ell \in \mathbb{N}$. For an $\ell$-tuple of variables $\tilde{x}=\left(x_{1}, \ldots, x_{\ell}\right)$ the $\ell$-flag polynomial is defined by

$$
\tilde{f}_{P}^{\ell}\left(x_{1}, \ldots, x_{\ell}\right)=\sum_{\tilde{s}} f_{\tilde{s}}(P) \tilde{x}^{\partial(\tilde{s})},
$$

where the sum is taken over all chains $\tilde{s}=\left(s_{1}, \ldots, s_{\ell}\right)$ with $0 \leq s_{1} \leq s_{2} \leq \cdots \leq s_{\ell} \leq d$ and $f_{\tilde{s}}(P)$ denotes the number of chains of faces $A_{1} \subseteq A_{2} \subseteq \cdots \subseteq A_{\ell}$ of $P$ with $\operatorname{dim}\left(A_{i}\right)=s_{i}$ for each $i \in\{1, \ldots, \ell\}$.

Note that each $\ell$-tuple $\tilde{a}$ of non-negative integers yields an $\ell$-tuple $\tilde{s}$ of increasing chain of integers $s_{1} \leq s_{2} \leq \cdots \leq s_{\ell}$ where $s_{i}=a_{1}+\cdots+a_{i}$ for each $i$ and so $\partial \tilde{s}=\tilde{a}$

The reasons why it might be worth working with the flag polynomial of a polytope is (i) its expression is simple for many basic polytopes like the simplex and hypercube and (ii) it behaves well under various natural constructions, the first of which is the Cartesian product.

Proposition 2.2 For polytopes $P$ and $Q$, a positive integer $\ell \in \mathbb{N}$ and $\tilde{x}=\left(x_{1}, \ldots, x_{\ell}\right)$ we have $\tilde{f}_{P \times Q}^{\ell}(\tilde{x})=\tilde{f}_{P}^{\ell}(\tilde{x}) \tilde{f}_{Q}^{\ell}(\tilde{x})$.

Proof. Any pair of chains $A_{1} \subseteq A_{2} \subseteq \cdots \subseteq A_{\ell} \subseteq P$ and $B_{1} \subseteq B_{2} \subseteq \cdots \subseteq B_{\ell} \subseteq Q$ yields a chain

$$
A_{1} \times B_{1} \subseteq A_{2} \times B_{2} \subseteq \cdots \subseteq A_{\ell} \times B_{\ell} \subseteq P \times Q
$$

of faces in the product $P \times Q$. Vice versa, any such chain of faces in $P \times Q$ of length $\ell$ yields a pair of chains of faces of length $\ell$ in $P$ and $Q$ respectively. Since the mapping $\tilde{s} \mapsto \partial \tilde{s}$ is linear the proposition follows.

Consider now the standard $(r-1)$-dimensional simplex $\Delta_{r-1}$. Let $0 \leq s_{1} \leq s_{2} \leq \cdots \leq s_{\ell} \leq r-1$ be an $\ell$-chain of nonnegative integers. Since any choice of $h+1$ vertices from the $r$ vertices of $\Delta_{r-1}$ 
is an $h$-face of $\Delta_{r-1}$, the number of chains $A_{1} \subseteq \cdots \subseteq A_{\ell}$ of faces of $\Delta_{r-1}$ with $\operatorname{dim}\left(A_{i}\right)=s_{i}$ for each $i$ is, by the product rule, given by

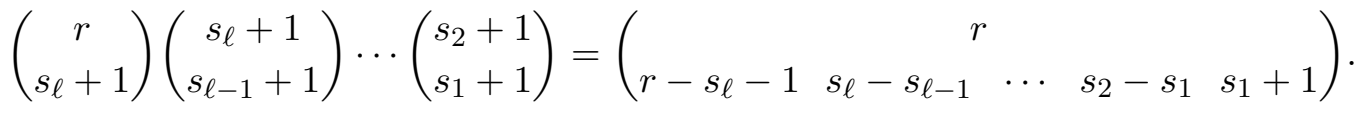

Since $\partial(\tilde{s})=\left(s_{1}, s_{2}-s_{1}, \ldots, s_{\ell}-s_{\ell-1}\right)$ we have the following.

Observation 2.3 For $\ell, r \in \mathbb{N}$ the $\ell$-flag polynomial of the standard simplex $\Delta_{r-1}$ is given by

$$
\tilde{f}_{\Delta_{r-1}}^{\ell}\left(x_{1}, \ldots, x_{\ell}\right)=\frac{\left(x_{1}+\cdots+x_{\ell}+1\right)^{r}-\left(x_{2}+\cdots+x_{\ell}+1\right)^{r}}{x_{1}} .
$$

By Proposition 2.2 and Observation 2.3 we have obtain the following.

Corollary 2.4 For $\ell \in \mathbb{N}$ and a polytope $P$ the $\ell$-flag polynomial of the prism $\operatorname{Pri}(P)=P \times \Delta_{1}$ of $P$ is given by

$$
\tilde{f}_{\operatorname{Pri}(P)}^{\ell}\left(x_{1}, \ldots, x_{\ell}\right)=\tilde{f}_{P}^{\ell}\left(x_{1}, \ldots, x_{\ell}\right)\left(2+x_{1}+2\left(x_{2}+\cdots+x_{\ell}\right)\right) .
$$

Since the $d$-dimensional hypercube $Q_{d}$ is given by $Q_{d}=\Delta_{1}{ }^{d}=\Delta_{1} \times \cdots \times \Delta_{1}$ (d-times), then we also obtain the following.

Corollary 2.5 For $\ell, d \in \mathbb{N}$ the $\ell$-flag polynomial of the hypercube $Q_{d}$ is given by

$$
\tilde{f}_{Q_{d}}^{\ell}\left(x_{1}, \ldots, x_{\ell}\right)=\left(2+x_{1}+2\left(x_{2}+\cdots+x_{\ell}\right)\right)^{d} .
$$

From this we see that the number of $\ell$-chains of faces of $Q_{d}$ is $(2 \ell+1)^{d}$. In particular, for $\ell=1$ we see that the total number of faces of $Q_{d}$ is $3^{d}$, the conjectured maximum number of faces of a centrally symmetric $d$-polytope [6].

Should one want to see the chains from the monomials more explicitly we have the following directly by Definition 2.1 of the flag polynomial.

Observation 2.6 Let $P$ be a polytope and $\ell \in \mathbb{N}$. If $\tilde{x}=\left(x_{1}, \ldots, x_{\ell}\right)$ and $\tilde{y}=\left(y_{1}, \ldots, y_{\ell}\right)$ are two tuples of variables such that $x_{i}=y_{i} y_{i+1} \cdots y_{\ell}$ for each $i=1, \ldots, \ell$, then the coefficient of $y_{1}^{s_{1}} y_{2}^{s_{2}} \cdots y_{\ell}^{s_{\ell}}$ in $\tilde{\phi}_{P}^{\ell}(\tilde{y}):=\tilde{f}_{P}^{\ell}(\tilde{x})$ is the number of chains of faces $A_{1} \subseteq A_{2} \subseteq \cdots \subseteq A_{\ell}$ of $P$ where $\operatorname{dim}\left(A_{i}\right)=s_{i}$ for each $i$.

REMARKS: (i) If for some reason one prefers to consider a polynomial directly corresponding to the flag vector (i.e. terms of the polynomial corresponding to strict chains of faces), then that can be obtained by the Inclusion/Exclusion principle: for each fixed $\ell$, the polynomial corresponding to the number of strict chains $A_{1} \subset \cdots \subset A_{\ell}$ of faces of a polynomial $P$ with $0 \leq \operatorname{dim}\left(A_{1}\right)<\cdots<$ $\operatorname{dim}\left(A_{\ell}\right) \leq \operatorname{dim}(P)$ is given by $\tilde{g}_{P}^{\ell}(\tilde{x})$ where

$$
\tilde{g}_{P}^{\ell}\left(x_{1}, \ldots, x_{\ell}\right)=\sum_{h=0}^{\ell-1}\left((-1)^{h} \sum_{2 \leq i_{1}<\cdots<i_{h} \leq \ell} \tilde{f}_{P}^{\ell-h}\left(\widehat{x_{i_{1}}}, \ldots, \widehat{x_{i_{h}}}\right)\right),
$$

where $\tilde{f}_{P}^{\ell-h}\left(\widehat{x_{i_{1}}}, \ldots, \widehat{x_{i_{h}}}\right)$ is the $(\ell-h)$-flag polynomial on the $(\ell-h)$-tuple obtained from $\left(x_{1}, \ldots, x_{\ell}\right)$ by removing the variables $x_{i_{j}}$ for $j=1, \ldots, h$. 
(ii) The number of terms in $\tilde{g}^{\ell}(\tilde{x})$ is $\left(\begin{array}{c}\operatorname{dim}(P)+1 \\ \ell\end{array}\right)$ whereas the number of terms in $\tilde{f}_{P}^{\ell}(\tilde{x})$ equals the number of solutions $\left(a_{1}, \ldots, a_{\ell}\right)$ to $a_{1}+\cdots+a_{\ell} \leq d=\operatorname{dim}(P)$ in nonnegative integers $a_{i} \geq 0$, namely $\left(\begin{array}{c}d+\ell \\ \ell\end{array}\right)$. Comparing the number of entries of the flag vector to the combined number of terms of all flag polynomials $\tilde{f}_{P}^{\ell}(\tilde{x})$ for $\ell=0,1, \ldots, d$, the combined number of terms equals

$$
\sum_{\ell=0}^{d}\left(\begin{array}{c}
d+\ell \\
\ell
\end{array}\right)=\left(\begin{array}{c}
2 d+1 \\
d+1
\end{array}\right) \sim \frac{2^{2 d+1}}{\sqrt{e \pi d}}
$$

not surprisingly an exponential expression which is considerably less than $\left(2^{d}\right)^{2}=4^{d}$, the square of the number of entries in the flag vector, but also more than $F_{d}$, the $d$-th Fibonacci number, the exact number of linearly independent terms [2].

In the next section we state Theorem 3.3, our main result, then parse through an elaborate example, before proving the main result in Section 4 .

\section{The flag polynomial of Minkowski sum of simplices}

If $\mathcal{F}$ consists of $k$ subsets of $[r]$ and is presented (merely for convenience) as an ordered tuple $\mathcal{F}=\left(F_{1}, F_{2}, \ldots, F_{k}\right)$, then for each $i \in[r]$ we let $N_{\mathcal{F}}(i)=\left\{j \in[k]: i \in F_{j}\right\}$. The following definition is from [8]:

Definition 3.1 For $k \in \mathbb{N}$ let $\mathcal{H}(k)=\left(H_{1}, \ldots, H_{k}\right)$ be the family of $k$ ordered subsets of [2 $\left.2^{k}-1\right]$ so that for $i \in\left\{1,2, \ldots, 2^{k}-1\right\}$ then $N_{\mathcal{H}(k)}(i)$ is the $i$-th nonempty subset of $[k]$ in the lexicographic order. Then the Minkowski sum of these $k$ simplices $P(k):=P_{\mathcal{H}(k)}$ is called the $k^{\text {th }}$ master polytope.

EXAmPle: For $k=2$ we have $\left[2^{k}-1\right]=[3]=\{1,2,3\}$ and the lexicographic ordering of the nonempty subsets of $[2]=\{1,2\}$ is given by $\{1,2\}>\{1\}>\{2\}$. By definition we must have $\mathcal{H}(k)=\mathcal{H}(2)=\left(H_{1}, H_{2}\right)$ where $H_{1}=\{1,2\}$ and $H_{2}=\{1,3\}$. In this case $P(2)=\Delta_{\{1,2\}}+\Delta_{\{1,3\}}$ is the 2-dimensional rhombus lying in the plane $x+y+z=2$ in $\mathbb{R}^{3}$ that is the convex hull of the points $(2,0,0),(1,1,0),(1,0,1)$ and $(0,1,1)$. In a similar fashion, for $k=3$ we have $\left[2^{k}-1\right]=[7]$ and the lexicographic ordering of the nonempty subsets of [3] is given by $\{1,2,3\}>\{1,2\}>\{1,3\}>$ $\{1\}>\{2,3\}>\{2\}>\{3\}$. By definition $\mathcal{H}(k)=\mathcal{H}(3)=\left(H_{1}, H_{2}, H_{3}\right)$ where $H_{1}=\{1,2,3,4\}$, $H_{2}=\{1,2,5,6\}$ and $H_{3}=\{1,3,5,7\}$. In this case $P(3)=\Delta_{H_{1}}+\Delta_{H_{2}}+\Delta_{H_{3}}$ is a 6-dimensional polytope in $\mathbb{R}^{7}$ with 41 vertices as verified by the computer program POLYMAKE [9] (see [8, Observations 2.13 and 2.16]).

Note that for a general $k \in \mathbb{N}$ then $P(k)=\sum_{i=1}^{k} \Delta_{H_{i}}$ where $\left|H_{i}\right|=2^{k-1}$ for each $i$. The master polytopes $P(k)$ for $k \geq 2$ are fundamental in understanding the overall structure of a general Minkowski sum of standard simplices, as we will see in Theorem 3.3. the main result in this section, where we express the flag polynomial of a general Minkowski sum of simplices. 
EXAmPLE: Since $P(2)$, the 2-dimensional rhombus, has the combinatorial structure of $\Delta_{1} \times \Delta_{1}$ we get by Proposition 2.2 and Observation 2.3 the following.

$$
\begin{aligned}
\tilde{f}_{P(2)}^{3}\left(x_{1}, x_{2}, x_{3}\right) & =\tilde{f}_{\Delta_{1} \times \Delta_{1}}^{3}\left(x_{1}, x_{2}, x_{3}\right) \\
& =\left[\tilde{f}_{\Delta_{1}}^{3}\left(x_{1}, x_{2}, x_{3}\right)\right]^{2} \\
& =\left(\frac{\left(x_{1}+x_{2}+x_{3}+1\right)^{2}-\left(x_{2}+x_{3}+1\right)^{2}}{x_{1}}\right)^{2} \\
& =\left(x_{1}+2 x_{2}+2 x_{3}+2\right)^{2},
\end{aligned}
$$

and hence by Observation 2.6

$$
\begin{aligned}
\tilde{\phi}_{P(2)}^{3}\left(y_{1}, y_{2}, y_{3}\right)= & \left(y_{1} y_{2} y_{3}+2 y_{2} y_{3}+2 y_{3}+2\right)^{2} \\
= & y_{1}^{2} y_{2}^{2} y_{3}^{2}+4 y_{1} y_{2}^{2} y_{3}^{2}+4 y_{1} y_{2} y_{3}^{2}+4 y_{2}^{2} y_{3}^{2}+ \\
& 4 y_{1} y_{2} y_{3}+8 y_{2} y_{3}^{2}+8 y_{2} y_{3}+4 y_{3}^{2}+8 y_{3}+4
\end{aligned}
$$

something which is evident by inspection.

From now on, we will assume $k \in \mathbb{N}$ fixed and we will always present our collection of subsets as an ordered $k$-tuple $\mathcal{F}=\left(F_{1}, F_{2}, \ldots, F_{k}\right)$ of $[r]$, and the Minkowski sum as

$$
P_{\mathcal{F}}=\sum_{F \in \mathcal{F}} \Delta_{F}=\Delta_{F_{1}}+\cdots+\Delta_{F_{k}} .
$$

We will assume that $\mathcal{F}$ covers all of $[r]$, that is $\bigcup_{F \in \mathcal{F}} F=[r]$, since otherwise we can simply restrict to this union. For a general Minkowski sum $P_{\mathcal{F}}$ of simplices as in (1), then for each $i \in\left[2^{k}-1\right]$ we let $R_{i}=\left\{j \in[r]: N_{\mathcal{F}}(j)=N_{\mathcal{H}(k)}(i)\right\}$ and $r_{i}=\left|R_{i}\right|$, so $r_{i}$ is the number of elements in the set $\left(\bigcap_{\ell \in N_{\mathcal{H}(k)}(i)} F_{\ell}\right) \cap\left(\bigcap_{\ell \notin N_{\mathcal{H}(k)}(i)} \overline{F_{\ell}}\right)$. Since $\mathcal{F}$ covers $[r]$ we have $r=r_{1}+\cdots+r_{2^{k}-1}$.

Note that for any polytope $P$ we have that $\tilde{f}_{P}^{\ell}\left(x_{1}, \ldots, x_{\ell-1}, 0\right)$ only has terms from $\tilde{f}_{P}^{\ell}\left(x_{1}, \ldots, x_{\ell}\right)$ where the coefficient for $x_{\ell}$ is zero. Hence, $\tilde{f}_{P}^{\ell}\left(x_{1}, \ldots, x_{\ell-1}, 0\right)$ is the generating function for the number of chains $Q_{1} \subseteq \cdots \subseteq Q_{\ell-1}=Q_{\ell}$ of faces of $P$, which is equivalent to the generating function for the number of chains $Q_{1} \subseteq \cdots \subseteq Q_{\ell-1}$ of faces of $P$.

Observation 3.2 For a polytope $P$ we have $\tilde{f}_{P}^{\ell}\left(x_{1}, \ldots, x_{\ell-1}, 0\right)=\tilde{f}_{P}^{\ell-1}\left(x_{1}, \ldots, x_{\ell-1}\right)$.

EXAmPle: Considering $P(2)$ again, we have from the above example that

$$
\tilde{f}_{P(2)}^{2}\left(x_{1}, x_{2}\right)=\tilde{f}_{P(2)}^{3}\left(x_{1}, x_{2}, 0\right)=\left(x_{1}+2 x_{2}+2\right)^{2} .
$$

Note that the total number of 2 -chains is the sum of the coefficients of $\tilde{f}_{P(2)}^{2}\left(x_{1}, x_{2}\right), 25$ altogether.

Conventions: (i) For a set of point $S \subseteq \mathbb{R}^{r}$ let $\operatorname{supp}(S)=\left\{i \in[r]: x_{i} \neq 0\right.$ for some $\tilde{x}=$ $\left.\left(x_{1}, \ldots, x_{r}\right) \in S\right\}$ be the support of $S$. (ii) For a face $A$ of $P$ let $\operatorname{dim}(A)$ denote the dimension of the face $A$. (iii) For a polytope $P$ we let $\mathbf{C}_{\ell}(P)$ denote the set of all $\ell$-chains $\tilde{A}=\left(A_{1}, \ldots, A_{\ell}\right)$ of faces of $P$ with $A_{1} \subseteq A_{2} \subseteq \cdots \subseteq A_{\ell}$. (iv) For each such $\tilde{A} \operatorname{let} \operatorname{dim}(\tilde{A})=\left(\operatorname{dim}\left(A_{1}\right), \ldots, \operatorname{dim}\left(A_{\ell}\right)\right)$ denote the chain of their corresponding dimensions.

With the above conventions in mind we have

$$
\partial(\operatorname{dim}(\tilde{A}))=\left(\operatorname{dim}\left(A_{1}\right), \operatorname{dim}\left(A_{2}\right)-\operatorname{dim}\left(A_{1}\right), \ldots, \operatorname{dim}\left(A_{\ell}\right)-\operatorname{dim}\left(A_{\ell-1}\right)\right) .
$$

We can now state the following main result in this section, whose proof is in Section 4 . 
Theorem 3.3 For $\ell \in \mathbb{N}$ and $\tilde{x}=\left(x_{1}, \ldots, x_{\ell}\right)$ the flag $\ell$-polynomial for a general Minkowski sum of simplices $P_{\mathcal{F}}=\sum_{F \in \mathcal{F}} \Delta_{F}=\Delta_{F_{1}}+\cdots+\Delta_{F_{k}}$ is given by

$$
\tilde{f}_{P_{\mathcal{F}}}^{\ell}\left(x_{1}, \ldots, x_{\ell}\right)=\sum_{\tilde{A} \in \mathbf{C}_{\ell}(P(k))}\left(\tilde{x}^{\partial(\operatorname{dim}(\tilde{A}))} \prod_{i=1}^{\ell}\left(\prod_{\alpha_{i} \in \operatorname{supp}\left(A_{i}\right) \backslash \operatorname{supp}\left(A_{i-1}\right)} \tilde{f}_{\Delta_{r_{\alpha_{i}}-1}}^{\ell-i+1}\left(x_{i}, \ldots, x_{\ell}\right)\right)\right) .
$$

In the formula above we interpret $\operatorname{supp}\left(A_{0}\right)=\emptyset$, since there is no $A_{0}$.

For each $\tilde{A} \in \mathbf{C}_{\ell}(P(k))$ let

$$
p_{\tilde{A}}\left(x_{1}, \ldots, x_{\ell}\right)=\tilde{x}^{\partial(\operatorname{dim}(\tilde{A}))} \prod_{i=1}^{\ell}\left(\prod_{\alpha_{i} \in \operatorname{supp}\left(A_{i}\right) \backslash \operatorname{supp}\left(A_{i-1}\right)} \tilde{f}_{\Delta_{r_{\alpha_{i}}-1}^{\ell-i+1}}\left(x_{i}, \ldots, x_{\ell}\right)\right) .
$$

For a concrete example we will parse through the very special case of Theorem 3.3 when $k=2$ and $\ell=2$. In this case $P_{\mathcal{F}}=\Delta_{F_{1}}+\Delta_{F_{2}}$ and $r_{1}=\left|F_{1} \cap F_{2}\right|, r_{2}=\left|F_{1} \backslash F_{2}\right|$ and $r_{3}=\left|F_{2} \backslash F_{1}\right|$. We will label the vertices of $P(2)$ as follows: $A=(2,0,0), B=(1,1,0), C=(1,0,1)$ and $D=(0,1,1)$. A 2-chain of faces of $P(2)$ will be given by $\tilde{A}=\left(A_{1}, A_{2}\right)$ where each face is presented as a string of the vertices whose convex combination forms the face. As noted in the last example there are 252 -chains of $P(2)$ and they can be grouped together by what $\operatorname{dim}(\tilde{A})=\left(\operatorname{dim}\left(A_{1}\right), \operatorname{dim}\left(A_{2}\right)\right)$ is as follows:

$$
\begin{array}{ll}
\operatorname{dim}(\tilde{A})=(0,0) \quad: \quad & (A, A),(B, B),(C, C),(D, D), \\
\operatorname{dim}(\tilde{A})=(1,1) \quad: \quad(A B, A B),(A C, A C),(B D, B D),(C D, C D), \\
\operatorname{dim}(\tilde{A})=(2,2) \quad: \quad(A B C D, A B C D), \\
\operatorname{dim}(\tilde{A})=(0,1) \quad: \quad(A, A B),(A, A C),(B, A B),(B, B D),(C, A C),(C, C D),(D, B D),(D, C D), \\
\operatorname{dim}(\tilde{A})=(1,2): \quad(A B, A B C D),(A C, A B C D),(B D, A B C D),(C D, A B C D), \\
\operatorname{dim}(\tilde{A})=(0,2): \quad(A, A B C D),(B, A B C D),(C, A B C D),(D, A B C D) .
\end{array}
$$

For $X \in\{A, B, C, D\}$ and $\tilde{A}=\left(A_{1}, A_{2}\right)=(X, X)$ we have

$$
\partial(\operatorname{dim}(\tilde{A}))=(\operatorname{dim}(X), \operatorname{dim}(X)-\operatorname{dim}(X))=(0,0),
$$

$\operatorname{supp}\left(A_{1}\right)=\operatorname{supp}(X), \operatorname{supp}\left(A_{2}\right) \backslash \operatorname{supp}\left(A_{1}\right)=\emptyset$, and hence

$$
p_{\tilde{A}}\left(x_{1}, x_{2}\right)=p_{(X, X)}\left(x_{1}, x_{2}\right)=\prod_{\alpha_{1} \in \operatorname{supp}(X)} \tilde{f}_{\Delta_{r_{\alpha_{1}}-1}}^{2}\left(x_{1}, x_{2}\right) .
$$

From this we obtain

$$
\begin{aligned}
p_{(A, A)}\left(x_{1}, x_{2}\right) & =\tilde{f}_{\Delta_{r_{1}-1}}^{2}\left(x_{1}, x_{2}\right), \\
p_{(B, B)}\left(x_{1}, x_{2}\right) & =\tilde{f}_{\Delta_{r_{1}-1}}^{2}\left(x_{1}, x_{2}\right) \tilde{f}_{\Delta_{r_{2}-1}}^{2}\left(x_{1}, x_{2}\right), \\
p_{(C, C)}\left(x_{1}, x_{2}\right) & =\tilde{f}_{\Delta_{r_{1}-1}}^{2}\left(x_{1}, x_{2}\right) \tilde{f}_{\Delta_{r_{3}-1}}^{2}\left(x_{1}, x_{2}\right), \\
p_{(D, D)}\left(x_{1}, x_{2}\right) & =\tilde{f}_{\Delta_{r_{2}-1}}^{2}\left(x_{1}, x_{2}\right) \tilde{f}_{\Delta_{r_{3}-1}}^{2}\left(x_{1}, x_{2}\right) .
\end{aligned}
$$

For $X, Y \in\{A, B, C, D\}$, and $\tilde{A}=\left(A_{1}, A_{2}\right)=(X Y, X Y)$ we have

$$
\partial(\operatorname{dim}(\tilde{A}))=(\operatorname{dim}(X Y), \operatorname{dim}(X Y)-\operatorname{dim}(X Y))=(1,0),
$$


$\operatorname{supp}\left(A_{1}\right)=\operatorname{supp}(X Y), \operatorname{supp}\left(A_{2}\right) \backslash \operatorname{supp}\left(A_{1}\right)=\emptyset$, and hence

$$
p_{\tilde{A}}\left(x_{1}, x_{2}\right)=p_{(X Y, X Y)}\left(x_{1}, x_{2}\right)=x_{1} \prod_{\alpha_{1} \in \operatorname{supp}(X Y)} \tilde{f}_{\Delta_{r_{\alpha_{1}}-1}}^{2}\left(x_{1}, x_{2}\right) .
$$

From this we obtain

$$
\begin{aligned}
p_{(A B, A B)}\left(x_{1}, x_{2}\right) & =x_{1} \tilde{f}_{\Delta_{r_{1}-1}}^{2}\left(x_{1}, x_{2}\right) \tilde{f}_{\Delta_{r_{2}-1}}^{2}\left(x_{1}, x_{2}\right), \\
p_{(A C, A C)}\left(x_{1}, x_{2}\right) & =x_{1} \tilde{f}_{\Delta_{r_{1}-1}}^{2}\left(x_{1}, x_{2}\right) \tilde{f}_{\Delta_{r_{3}-1}}^{2}\left(x_{1}, x_{2}\right), \\
p_{(B D, B D)}\left(x_{1}, x_{2}\right) & =x_{1} \tilde{f}_{\Delta_{r_{1}-1}}^{2}\left(x_{1}, x_{2}\right) \tilde{f}_{\Delta_{r_{2}-1}}^{2}\left(x_{1}, x_{2}\right) \tilde{f}_{\Delta_{r_{3}-1}}^{2}\left(x_{1}, x_{2}\right), \\
p_{(C D, C D)}\left(x_{1}, x_{2}\right) & =x_{1} \tilde{f}_{\Delta_{r_{1}-1}}^{2}\left(x_{1}, x_{2}\right) \tilde{f}_{\Delta_{r_{2}-1}}^{2}\left(x_{1}, x_{2}\right) \tilde{f}_{\Delta_{r_{3}-1}}^{2}\left(x_{1}, x_{2}\right) .
\end{aligned}
$$

For $\tilde{A}=\left(A_{1}, A_{2}\right)=(A B C D, A B C D)$ we have

$$
\partial(\operatorname{dim}(\tilde{A}))=(\operatorname{dim}(A B C D), \operatorname{dim}(A B C D)-\operatorname{dim}(A B C D))=(2,0),
$$

$\operatorname{supp}\left(A_{1}\right)=\operatorname{supp}(A B C D)=\{1,2,3\}, \operatorname{supp}\left(A_{2}\right) \backslash \operatorname{supp}\left(A_{1}\right)=\emptyset$, and hence

$$
p_{\tilde{A}}\left(x_{1}, x_{2}\right)=p_{(A B C D, A B C D)}\left(x_{1}, x_{2}\right)=x_{1}^{2} \prod_{\alpha_{1} \in \operatorname{supp}(A B C D)} \tilde{f}_{\Delta_{r_{\alpha_{1}}-1}}^{2}\left(x_{1}, x_{2}\right) .
$$

From this we obtain

$$
p_{(A B C D, A B C D)}\left(x_{1}, x_{2}\right)=x_{1}^{2} \tilde{f}_{\Delta_{r_{1}-1}}^{2}\left(x_{1}, x_{2}\right) \tilde{f}_{\Delta_{r_{2}-1}}^{2}\left(x_{1}, x_{2}\right) \tilde{f}_{\Delta_{r_{3}-1}}^{2}\left(x_{1}, x_{2}\right) .
$$

For $X, Y \in\{A, B, C, D\}$, and $\tilde{A}=\left(A_{1}, A_{2}\right)=(X, X Y)$ we have

$$
\partial(\operatorname{dim}(\tilde{A}))=(\operatorname{dim}(X), \operatorname{dim}(X Y)-\operatorname{dim}(X))=(0,1),
$$

$\operatorname{supp}\left(A_{1}\right)=\operatorname{supp}(X), \operatorname{supp}\left(A_{2}\right) \backslash \operatorname{supp}\left(A_{1}\right)=\operatorname{supp}(X Y) \backslash \operatorname{supp}(X)$, and hence

$$
p_{\tilde{A}}\left(x_{1}, x_{2}\right)=p_{(X, X Y)}\left(x_{1}, x_{2}\right)=x_{2} \prod_{\alpha_{1} \in \operatorname{supp}(X)} \tilde{f}_{\Delta_{r_{\alpha_{1}}-1}}^{2}\left(x_{1}, x_{2}\right) \prod_{\alpha_{2} \in \operatorname{supp}(X Y) \backslash \operatorname{supp}(X)} \tilde{f}_{\Delta_{r_{\alpha_{2}}-1}}^{1}\left(x_{2}\right) .
$$

From this we obtain

$$
\begin{aligned}
p_{(A, A B)}\left(x_{1}, x_{2}\right) & =x_{2} \tilde{f}_{\Delta_{r_{1}-1}}^{2}\left(x_{1}, x_{2}\right) \tilde{f}_{\Delta_{r_{2}-1}}^{1}\left(x_{2}\right), \\
p_{(A, A C)}\left(x_{1}, x_{2}\right) & =x_{2} \tilde{f}_{\Delta_{r_{1}-1}}^{2}\left(x_{1}, x_{2}\right) \tilde{f}_{\Delta_{r_{3}-1}}^{1}\left(x_{2}\right), \\
p_{(B, A B)}\left(x_{1}, x_{2}\right) & =x_{2} \tilde{f}_{\Delta_{r_{1}-1}}^{2}\left(x_{1}, x_{2}\right) \tilde{f}_{\Delta_{r_{2}-1}}^{2}\left(x_{1}, x_{2}\right) \\
p_{(B, B D)}\left(x_{1}, x_{2}\right) & =x_{2} \tilde{f}_{\Delta_{r_{1}-1}}^{2}\left(x_{1}, x_{2}\right) \tilde{f}_{\Delta_{r_{2}-1}}^{2}\left(x_{1}, x_{2}\right) \tilde{f}_{\Delta_{r_{3}-1}}^{1}\left(x_{2}\right), \\
p_{(C, A C)}\left(x_{1}, x_{2}\right) & =x_{2} \tilde{f}_{\Delta_{r_{1}-1}}^{2}\left(x_{1}, x_{2}\right) \tilde{f}_{\Delta_{r_{3}-1}}^{2}\left(x_{1}, x_{2}\right) \\
p_{(C, C D)}\left(x_{1}, x_{2}\right) & =x_{2} \tilde{f}_{\Delta_{r_{1}-1}}^{2}\left(x_{1}, x_{2}\right) \tilde{f}_{\Delta_{r_{3}-1}}^{2}\left(x_{1}, x_{2}\right) \tilde{f}_{\Delta_{r_{2}-1}}^{1}\left(x_{2}\right), \\
p_{(D, B D)}\left(x_{1}, x_{2}\right) & =x_{2} \tilde{f}_{\Delta_{r_{2}-1}}^{2}\left(x_{1}, x_{2}\right) \tilde{f}_{\Delta_{r_{3}-1}}^{2}\left(x_{1}, x_{2}\right) \tilde{f}_{\Delta_{r_{1}-1}}^{1}\left(x_{2}\right), \\
p_{(D, C D)}\left(x_{1}, x_{2}\right) & =x_{2} \tilde{f}_{\Delta_{r_{2}-1}}^{2}\left(x_{1}, x_{2}\right) \tilde{f}_{\Delta_{r_{3}-1}}^{2}\left(x_{1}, x_{2}\right) \tilde{f}_{\Delta_{r_{1}-1}}^{1}\left(x_{2}\right) .
\end{aligned}
$$


For $X Y \in\{A B, A C, B D, C D\}$, and $\tilde{A}=\left(A_{1}, A_{2}\right)=(X Y, A B C D)$ we have

$$
\partial(\operatorname{dim}(\tilde{A}))=(\operatorname{dim}(X Y), \operatorname{dim}(A B C D)-\operatorname{dim}(X Y))=(1,1),
$$

$\operatorname{supp}\left(A_{1}\right)=\operatorname{supp}(X Y), \operatorname{supp}\left(A_{2}\right) \backslash \operatorname{supp}\left(A_{1}\right)=\operatorname{supp}(A B C D) \backslash \operatorname{supp}(X Y)=\{1,2,3\} \backslash \operatorname{supp}(X Y)$ and hence

$$
p_{\tilde{A}}\left(x_{1}, x_{2}\right)=p_{(X Y, A B C D)}\left(x_{1}, x_{2}\right)=x_{1} x_{2} \prod_{\alpha_{1} \in \operatorname{supp}(X Y)} \tilde{f}_{\Delta_{r_{\alpha_{1}-1}}^{2}}^{2}\left(x_{1}, x_{2}\right) \prod_{\alpha_{2} \in\{1,2,3\} \backslash \operatorname{supp}(X Y)} \tilde{f}_{\Delta_{r_{\alpha_{2}}-1}}\left(x_{2}\right) .
$$

From this we obtain

$$
\begin{aligned}
p_{(A B, A B C D)}\left(x_{1}, x_{2}\right) & =x_{1} x_{2} \tilde{f}_{\Delta_{r_{1}-1}}^{2}\left(x_{1}, x_{2}\right) \tilde{f}_{\Delta_{r_{2}-1}}^{2}\left(x_{1}, x_{2}\right) \tilde{f}_{\Delta_{r_{3}-1}}^{1}\left(x_{2}\right), \\
p_{(A C, A B C D)}\left(x_{1}, x_{2}\right) & =x_{1} x_{2} \tilde{f}_{\Delta_{r_{1}-1}}^{2}\left(x_{1}, x_{2}\right) \tilde{f}_{\Delta_{r_{3}-1}}^{2}\left(x_{1}, x_{2}\right) \tilde{f}_{\Delta_{r_{2}-1}}^{1}\left(x_{2}\right), \\
p_{(B D, A B C D)}\left(x_{1}, x_{2}\right) & =x_{1} x_{2} \tilde{f}_{\Delta_{r_{1}-1}}^{2}\left(x_{1}, x_{2}\right) \tilde{f}_{\Delta_{r_{2}-1}}^{2}\left(x_{1}, x_{2}\right) \tilde{f}_{\Delta_{r_{3}-1}}^{2}\left(x_{1}, x_{2}\right), \\
p_{(C D, A B C D)}\left(x_{1}, x_{2}\right) & =x_{1} x_{2} \tilde{f}_{\Delta_{r_{1}-1}}^{2}\left(x_{1}, x_{2}\right) \tilde{f}_{\Delta_{r_{2}-1}}^{2}\left(x_{1}, x_{2}\right) \tilde{f}_{\Delta_{r_{3}-1}}^{2}\left(x_{1}, x_{2}\right) .
\end{aligned}
$$

Finally, for $X \in\{A, B, C, D\}$, and $\tilde{A}=\left(A_{1}, A_{2}\right)=(X, A B C D)$ we have

$$
\partial(\operatorname{dim}(\tilde{A}))=(\operatorname{dim}(X), \operatorname{dim}(A B C D)-\operatorname{dim}(X))=(0,2),
$$

$\operatorname{supp}\left(A_{1}\right)=\operatorname{supp}(X), \operatorname{supp}\left(A_{2}\right) \backslash \operatorname{supp}\left(A_{1}\right)=\{1,2,3\} \backslash \operatorname{supp}(X)$, and hence

$$
p_{\tilde{A}}\left(x_{1}, x_{2}\right)=p_{(X, A B C D)}\left(x_{1}, x_{2}\right)=x_{2}^{2} \prod_{\alpha_{1} \in \operatorname{supp}(X)} \tilde{f}_{\Delta_{r_{\alpha_{1}}-1}}^{2}\left(x_{1}, x_{2}\right) \prod_{\alpha_{2} \in\{1,2,3\} \backslash \operatorname{supp}(X)} \tilde{f}_{\Delta_{r_{\alpha_{2}}-1}}^{1}\left(x_{2}\right) .
$$

From this we obtain

$$
\begin{aligned}
& p_{(A, A B C D)}\left(x_{1}, x_{2}\right)=x_{2}^{2} \tilde{f}_{\Delta_{r_{1}-1}}^{2}\left(x_{1}, x_{2}\right) \tilde{f}_{\Delta_{r_{2}-1}}^{1}\left(x_{2}\right) \tilde{f}_{\Delta_{r_{3}-1}}^{1}\left(x_{2}\right), \\
& p_{(B, A B C D)}\left(x_{1}, x_{2}\right)=x_{2}^{2} \tilde{f}_{\Delta_{r_{1}-1}}^{2}\left(x_{1}, x_{2}\right) \tilde{f}_{\Delta_{r_{2}-1}}^{2}\left(x_{1}, x_{2}\right) \tilde{f}_{\Delta_{r_{3}-1}}^{1}\left(x_{2}\right), \\
& p_{(C, A B C D)}\left(x_{1}, x_{2}\right)=x_{2}^{2} \tilde{f}_{\Delta_{r_{1}-1}}^{2}\left(x_{1}, x_{2}\right) \tilde{f}_{\Delta_{r_{3}-1}}^{2}\left(x_{1}, x_{2}\right) \tilde{f}_{\Delta_{r_{2}-1}}^{1}\left(x_{2}\right), \\
& p_{(D, A B C D)}\left(x_{1}, x_{2}\right)=x_{2}^{2} \tilde{f}_{\Delta_{r_{2}-1}}^{2}\left(x_{1}, x_{2}\right) \tilde{f}_{\Delta_{r_{3}-1}}^{2}\left(x_{1}, x_{2}\right) \tilde{f}_{\Delta_{r_{1}-1}}^{1}\left(x_{2}\right) .
\end{aligned}
$$

Hence, collecting all the data for $P_{\mathcal{F}}=\Delta_{F_{1}}+\Delta_{F_{2}}$ with $r_{1}=\left|F_{1} \cap F_{2}\right|, r_{2}=\left|F_{1} \backslash F_{2}\right|$ and $r_{3}=\left|F_{2} \backslash F_{1}\right|$ we have by Theorem 3.3 that

$$
\tilde{f}_{P_{\mathcal{F}}}^{2}\left(x_{1}, x_{2}\right)=\sum_{\tilde{A} \in \mathbf{C}_{2}(P(2))} p_{\tilde{A}}\left(x_{1}, x_{2}\right),
$$

where the sum is taken over the 25 different 2-chains $\tilde{A}$ given in (2). Substituting we obtain the following.

Corollary 3.4 If $F_{1}, F_{2} \subseteq[r]$ and $r_{1}=\left|F_{1} \cap F_{2}\right|, r_{2}=\left|F_{1} \backslash F_{2}\right|$ and $r_{3}=\left|F_{2} \backslash F_{1}\right|$ then for $P_{\mathcal{F}}=\Delta_{F_{1}}+\Delta_{F_{2}}$ We have

$$
\begin{aligned}
\tilde{f}_{P_{\mathcal{F}}}^{2}\left(x_{1}, x_{2}\right)= & \left(2 x_{1}+x_{1}^{2}+2 x_{1} x_{2}\right) \tilde{f}_{1}^{2} \tilde{f}_{2}^{2} \tilde{f}_{3}^{2}+\left(x_{2}+x_{1} x_{2}+x_{2}^{2}\right)\left(\tilde{f}_{1}^{2} \tilde{f}_{2}^{2} \tilde{f}_{3}^{1}+\tilde{f}_{1}^{2} \tilde{f}_{3}^{2} \tilde{f}_{2}^{1}\right) \\
& +\left(2 x_{2}+x_{2}^{2}\right) \tilde{f}_{2}^{2} \tilde{f}_{3}^{2} \tilde{f}_{1}^{1}+x_{2}^{2} \tilde{f}_{1}^{2} \tilde{f}_{2}^{1} \tilde{f}_{3}^{1} \\
& +\left(1+x_{1}+x_{2}\right)\left(\tilde{f}_{1}^{2} \tilde{f}_{2}^{2}+\tilde{f}_{1}^{2} \tilde{f}_{3}^{2}\right)+x_{2}\left(\tilde{f}_{1}^{2} \tilde{f}_{2}^{1}+\tilde{f}_{1}^{2} \tilde{f}_{3}^{1}\right)+\tilde{f}_{2}^{2} \tilde{f}_{3}^{2}+\tilde{f}_{1}^{2},
\end{aligned}
$$

where $\tilde{f}_{i}^{2}=\tilde{f}_{\Delta_{r_{i}-1}}^{2}\left(x_{1}, x_{2}\right)$ and $\tilde{f}_{i}^{1}=\tilde{f}_{\Delta_{r_{i}-1}}^{1}\left(x_{2}\right)$ for $i \in\{1,2,3\}$ are as in Observation 2.3. 


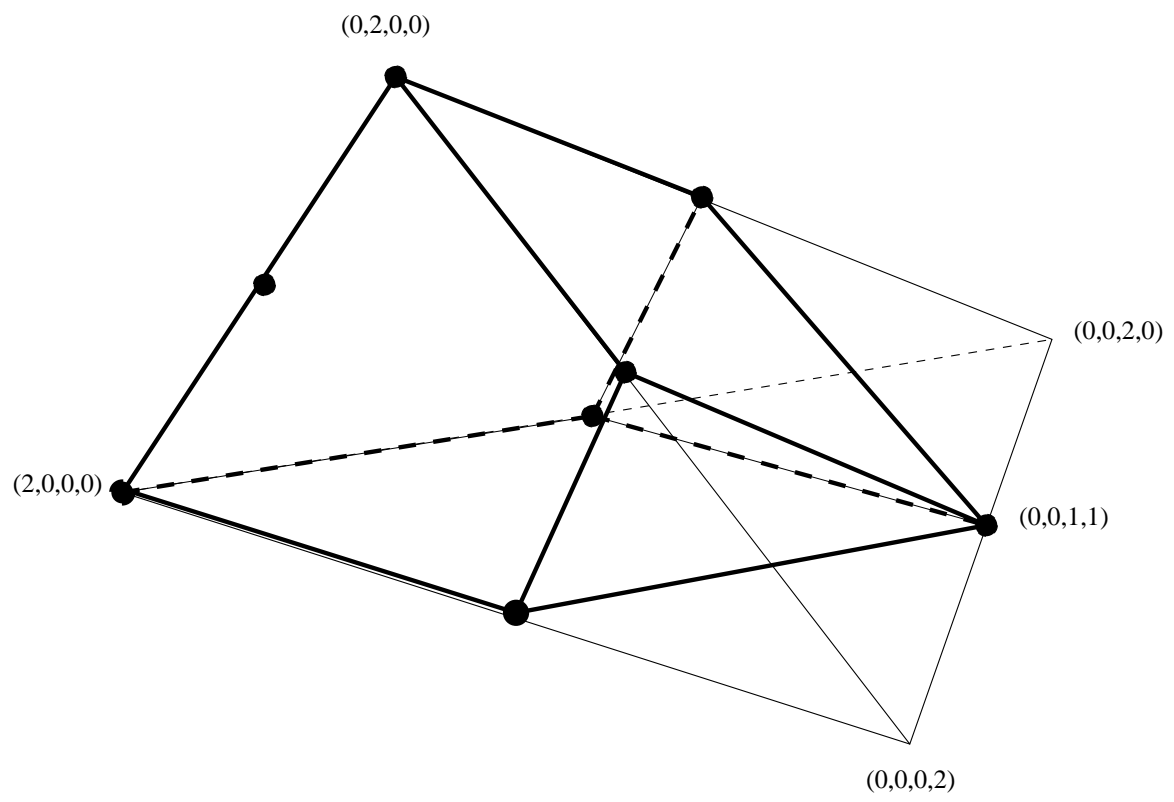

Figure 1: $\Delta_{F_{1}}+\Delta_{F_{2}}$ where $F_{1}=\{1,2,3\}$ and $F_{2}=\{1,2,4\}$.

ExAmple: For $F_{1}=\{1,2,3\}$ and $F_{2}=\{1,2,4\}$ we have $r_{1}=2$ and $r_{2}=r_{3}=1$ and we get in this case that

$$
\tilde{f}_{\Delta_{F_{1}}+\Delta_{F_{2}}}^{2}\left(x_{1}, x_{2}\right)=7+11 x_{1}+6 x_{1}^{2}+x_{1}^{3}+22 x_{2}+22 x_{1} x_{2}+6 x_{1}^{2} x_{2}+22 x_{2}^{2}+11 x_{1} x_{2}^{2}+7 x_{2}^{3},
$$

something that can be seen by inspection, since the Minkowski sum $\Delta_{F_{1}}+\Delta_{F_{2}}$ in this case has the same combinatorial structure as a cube where one side/edge has been contracted to a vertex. The polytope $\Delta_{F_{1}}+\Delta_{F_{2}}$ has been drawn in Figure 1 .

EXAMPLE: Using the setup from Corollary 3.4 above, we note that the total number of vertices of $P_{\mathcal{F}}=\Delta_{F_{1}}+\Delta_{F_{2}}$ is the number of $(0,0)$ 2-chains of faces, which is precisely the constant term in $\tilde{f}_{P_{\mathcal{F}}}^{2}\left(x_{1}, x_{2}\right)$ given by $\tilde{f}_{P_{\mathcal{F}}}^{2}(0,0)$. Since $\tilde{f}_{\Delta_{r_{i}-1}}^{2}\left(x_{1}, x_{2}\right)=\left(\left(x_{1}+x_{2}+1\right)^{r_{i}}-\left(x_{2}+1\right)^{r_{i}}\right) / x_{1}$ and $\tilde{f}_{\Delta_{r_{i}-1}}^{1}\left(x_{2}\right)=\left(\left(x_{2}+1\right)^{r_{i}}-1\right) / x_{2}$ by Observation 2.3 , we get $\tilde{f}_{\Delta_{r_{i}-1}}^{2}(0,0)=\tilde{f}_{\Delta_{r_{i}-1}}^{1}(0)=r_{i}$ for each $i$, and hence by Corollary 3.4 the number of vertices is in this case

$$
\tilde{f}_{P_{\mathcal{F}}}^{2}(0,0)=r_{1} r_{2}+r_{1} r_{3}+r_{2} r_{3}+r_{1} .
$$

We will revisit this example in Section 5 ,

\section{Generalizing the free join of polytopes}

In this section we describe how viewing the free join of two polytopes from a different angle, naturally generalizes to an action of a polytope on a set of polytopes. We then present the $\ell$-flag polynomial for the resulting polytope and finally apply that to the Minkowski sum of simplices.

For polytopes $P$ and $Q$ recall that the free join (or just the join as it is sometimes called when there is no danger of ambiguity [10] $P * Q$ is a polytope that is obtained by positioning $P$ and $Q$ 
into skew affine subspaces and then take the convex hull $\operatorname{conv}(P, Q)$. The faces of $P * Q$ are given by (i) type $P$ : the faces of $P$, (ii) type $Q$ : the faces of $Q$ and (iii) type $P Q$ : the disjoint union $A \cup B$ where $A$ is a face of $P$ and $B$ a face of $Q$. The dimension of a face of type $P$ or $Q$ is equal to its dimension as a face in $P$ or $Q$, but the dimension of a face $A \cup B$ of type $P Q$ is equal to $\operatorname{dim}(A)+\operatorname{dim}(B)+1$ because of the convex combination of points of $A$ and points of $B$. The three types of faces of $P * Q$ is a partition of its faces. The free join of a polytope $P$ and a singleton $\tilde{x}_{0}$ is called the pyramid over $P$ and denoted $\operatorname{Pyr}(P)$ [10].

For a chain $C_{1} \subseteq C_{2} \subseteq \cdots \subseteq C_{\ell}$ of faces of $P * Q$ there are five types of chains depending on the type of the starting and terminal face: types $(P, P),(P, P Q),(P Q, P Q),(Q, P Q)$ and $(Q, Q)$. The $\ell$-flag polynomial for type $(P, P)$ and $(Q, Q)$ chains is $\tilde{f}_{P}^{\ell}(\tilde{x})$ and $\tilde{f}_{Q}^{\ell}(\tilde{x})$ respectively. The $\ell$ flag polynomial for a type $(P Q, P Q)$ chain is the same as for $P \times Q$ except for the fact that the dimension of each face $A \cup B$ is one more that that of $A \times B$. Hence, the $\ell$-flag polynomial must be shifted upward by one at each level of the chain. By definition of the flag polynomial, that is obtained by multiplying by the the first variable $x_{1}$ and we obtain $x_{1} \tilde{f}_{P}^{\ell}(\tilde{x}) \tilde{f}_{Q}^{\ell}(\tilde{x})$. For a type $(P, P Q)$ chain, there are $\ell-1$ possible places in the chain where we encounter the first type $P Q$ face, say at step $i \in\{2, \ldots, \ell\}$. Each such chain is determined by an $\ell$-chain of faces in $P$ (the $A_{i}$ s) and an additional $(\ell-i+1)$-chain in $Q$ (the $B_{i}$ s) and has the form

$$
A_{1} \subseteq \cdots \subseteq A_{i-1} \subseteq A_{i} * B_{i} \subseteq \cdots \subseteq A_{\ell} * B_{\ell}
$$

Again, since the dimension of each $A_{j} * B_{j}$ is one more than that of $A_{j} \times B_{j}$, we must shift the dimension upward by one from and including at level $i$, which is obtained by multiplying by $x_{i}$. Hence, the $\ell$-flag polynomial for all chains of the form (3) is given by $x_{i} \tilde{f}_{P}^{\ell}(\tilde{x}) \tilde{f}_{Q}^{\ell-i+1}\left(x_{i}, \ldots, x_{\ell}\right)$. Since the case for type $(Q, P Q)$ chains is treated in the same way we obtain the following.

Proposition 4.1 For polytopes $P$ and $Q$ and a positive integer $\ell \in \mathbb{N}$, the $\ell$-flag polynomial for the free join $P * Q$ is given by

$$
\begin{aligned}
\tilde{f}_{P * Q}^{\ell}(\tilde{x}) & =\tilde{f}_{P}^{\ell}(\tilde{x})\left(1+\sum_{i=2}^{\ell} x_{i} \tilde{f}_{Q}^{\ell-i+1}\left(x_{i}, \ldots, x_{\ell}\right)\right) \\
& +\tilde{f}_{Q}^{\ell}(\tilde{x})\left(1+\sum_{i=2}^{\ell} x_{i} \tilde{f}_{P}^{\ell-i+1}\left(x_{i}, \ldots, x_{\ell}\right)\right) \\
& +x_{1} \tilde{f}_{P}^{\ell}(\tilde{x}) \tilde{f}_{Q}^{\ell}(\tilde{x}) .
\end{aligned}
$$

Note that for the empty polytope $\emptyset$ we have $P * \emptyset=P$ and $\emptyset * Q=Q$. Also, for each $\ell$ we have $\tilde{f}_{\emptyset}^{\ell}(\tilde{x})=0$.

When $Q$ is a singleton $Q=\Delta_{0}$, then $\tilde{f}_{Q}^{\ell}(\tilde{x})=1$ for each $\ell$, and hence we obtain the following.

Corollary 4.2 For a polytope $P$ and $\ell \in \mathbb{N}$, the $\ell$-flag polynomial for the pyramid $\operatorname{Pyr}(P)$ over $P$ is given by

$$
\tilde{f}_{\mathrm{Pyr}(P)}^{\ell}(\tilde{x})=\tilde{f}_{P}^{\ell}(\tilde{x})\left(1+x_{1}+\cdots+x_{\ell}\right)+1+\sum_{i=2}^{\ell} x_{i} \tilde{f}_{P}^{\ell-i+1}\left(x_{i}, \ldots, x_{\ell}\right) .
$$

REMARK: For a $d$-polytope the $d$-flag-polynomial contains all the information of the flag vector and the cd-index of $P$ with many redundancies (i.e. the coefficient of different monomials of the flag polynomial will count the same number of strict chains). Working with the most compact encoding 
of the flag vector, elegant formulae for computing the cd-indices of the prism $\operatorname{Pri}(P)=P \times \Delta_{1}$ and the pyramid $\operatorname{Pyr}(P)=P * \Delta_{0}$ in terms of the cd-index $\Psi(P)$ of $P$ and some linear derivations $\mathbb{R}\langle\mathbf{c}, \mathbf{d}\rangle \rightarrow \mathbb{R}\langle\mathbf{c}, \mathbf{d}\rangle$ are given in [4]. But even with these nice recursions, it is hard to obtain explicit formulae for the cd-index of specific polytopes, like the simplex $\Delta_{d}=\operatorname{Pyr}\left(\Delta_{d-1}\right)$, largely since derivations on non-commuting monomials in $\mathbb{R}\langle\mathbf{c}, \mathbf{d}\rangle$ expand exponentially in repeated steps and do not simplify easily. These recursions are generalized to a recursion to compute the cd-indices for the free join and the Cartesian product in [5].

We now generalize the idea of the free join and investigate the effect on the corresponding flag polynomial from Proposition 4.1. First note that a concrete geometric realization of the free join $Q_{1} * Q_{2}$ can be obtained by an action $\left(Q_{1}, Q_{2}\right) \mapsto \Delta_{1}\left(Q_{1}, Q_{2}\right)$ of the 1-simplex $\Delta_{1}$ on the polytopes $Q_{1}$ and $Q_{2}$. Here $\Delta_{1}$ is the standard geometric 1-dimensional simplex $\Delta_{[2]}=\left\{\left(x_{1}, x_{2}\right): x_{1}, x_{2} \geq\right.$ $\left.0, x_{1}+x_{2}=1\right\}$, and we attach $Q_{1}$ to one endvertex $(1,0)$ and $Q_{2}$ to the other $(0,1)$. Here we also choose an embedding of each $Q_{i}$ in $\left\{\tilde{x}_{i} \in \mathbb{R}^{d_{i}+1}: \sum x_{i j}=1\right\}$ where $d_{i}=\operatorname{dim}\left(Q_{i}\right)$, and the action is given by

$$
\Delta_{1}\left(Q_{1}, Q_{2}\right):=\left\{\left(t_{1} \tilde{x}_{1}, t_{2} \tilde{x}_{2}\right) \in \mathbb{R}^{d_{1}+d_{2}+2}: t_{1}, t_{2} \geq 0, t_{1}+t_{2}=1, \tilde{x}_{1} \in Q_{1}, \tilde{x}_{2} \in Q_{2}\right\} .
$$

Note that for a nonempty polytope $Q$ we have $\Delta_{1}(Q, \emptyset)=\Delta_{1}(\emptyset, Q)=Q$. However, if both $Q_{1}$ and $Q_{2}$ are nonempty, then every face of $\Delta_{1}\left(Q_{1}, Q_{2}\right)$ has the form $A\left(F_{1}, F_{2}\right)$ where each $F_{i}$ is a face of $Q_{i}$ or $Q_{i}$ itself, and $A$ is a face of $\Delta_{1}$ or $\Delta_{1}$ itself.

Each $\ell$-chain of faces of $\Delta_{1}\left(Q_{1}, Q_{2}\right)$ yields, by projection, an $\ell$-chain of faces $A_{1} \subseteq \cdots \subseteq A_{\ell}$ of $\Delta_{1}$, and hence we have a partition of the $\ell$-chains of faces of $\Delta_{1}\left(Q_{1}, Q_{2}\right)$ where each part consists of the chains that yield the same chain of $\Delta_{1}$. Note that each proper face $A$ of $\Delta_{1}$ has $\operatorname{supp}(A) \in\{\{1\},\{2\},\{1,2\}\}$ and hence each $\ell$-chain $\tilde{A}$ of faces of $\Delta_{1}$ has $\tilde{x}^{\partial(\operatorname{dim}(\tilde{A}))} \in\left\{1, x_{1}, \ldots, x_{\ell}\right\}$. Writing $Q_{1}$ for $P$ and $Q_{2}$ for $Q$ in Proposition 4.1, the $\ell$-flag polynomial in Proposition 4.1 for $Q_{1} * Q_{2}$ equals that of is geometric realization $\Delta_{1}\left(Q_{1}, Q_{2}\right)$, and we obtain

$$
\tilde{f}_{Q_{1} * Q_{2}}^{\ell}\left(x_{1}, \ldots, x_{\ell}\right)=\sum_{\tilde{A} \in \mathbf{C}_{\ell}\left(\Delta_{1}\right)}\left(\tilde{x}^{\partial(\operatorname{dim}(\tilde{A}))} \prod_{i=1}^{\ell}\left(\prod_{\alpha_{i} \in \operatorname{supp}\left(A_{i}\right) \backslash \operatorname{supp}\left(A_{i-1}\right)} \tilde{f}_{Q_{\alpha_{i}}^{\ell-i+1}}\left(x_{i}, \ldots, x_{\ell}\right)\right)\right) .
$$

Conventions: (i) Call an embedding of a polytope $P$ in $\mathbb{R}^{\operatorname{dim}(P)+1}$ simplicial if $P \subseteq \Delta_{\operatorname{dim}(P)}=$ $\Delta_{[\operatorname{dim}(P)+1]}$. (ii) By a full face of a polytope we will mean either a face or the entire polytope itself.

The repeated free $m$-join $Q_{1} * \cdots * Q_{m}$ of polytopes $Q_{i}$ s is well defined since the operation $*$ is associative and commutative, and it has the empty polytope $\emptyset$ as the identity element. This operation $*$ makes the collection $\mathcal{Q}$ of all polytopes $Q$ a commutative $\operatorname{semigroup}(\mathcal{Q}, *)$.

Observation 4.3 Let $Q_{1}, \ldots, Q_{m}$ be polytopes (the empty polytope $\emptyset$ is allowed). The $m$-fold free join $Q_{1} * \cdots * Q_{m}$ is a polytope of dimension $m-1+\sum_{i=1}^{m} \operatorname{dim}\left(Q_{i}\right)$ in which each full face has the the form $F_{1} * \cdots * F_{m}$ where each $F_{i}$ is a full face of $Q_{i}$ or the empty face. As $\operatorname{dim}(\emptyset)=-1$, the dimension of each face is given by $\operatorname{dim}\left(F_{1} * \cdots * F_{m}\right)=m-1+\sum_{i=1}^{m} \operatorname{dim}\left(F_{i}\right)$.

One nice advantage of (4) over the formula in Proposition 4.1 is that it generalizes naturally in light of Observation 4.3. This is since a geometric realization of the $m$-fold free join $Q_{1} * \cdots * Q_{m}$ can, as above, be obtained by an action $\left(Q_{1}, \ldots, Q_{m}\right) \mapsto \Delta_{m-1}\left(Q_{1}, \ldots, Q_{m}\right)$ of the standard $(m-1)$ simplex $\Delta_{m-1}$ on $Q_{1}, \ldots, Q_{m}$. We attach each $Q_{i}$ to an endvertex $\tilde{e}_{i}$ of $\Delta_{m-1}$ and so

$$
\Delta_{m-1}\left(Q_{1}, \ldots, Q_{m}\right):=\left\{\left(t_{1} \tilde{x}_{1}, \ldots, t_{m} \tilde{x}_{m}\right) \in \mathbb{R}^{m+\sum d_{i}}: \sum_{i} t_{i}=1, t_{i} \geq 0, \tilde{x}_{i} \in Q_{i} \text { for each } i\right\} .
$$


Here we do assume each $Q_{i}$ to be a nonempty polytope, and so each full face of $\Delta_{m-1}\left(Q_{1}, \ldots, Q_{m}\right)$ has the form $A\left(F_{1}, \ldots, F_{m}\right)$ where $A$ is a full face of $\Delta_{m-1}$ and each $F_{i}$ is a full face of $Q_{i}$.

As $\Delta_{m-1}\left(Q_{1}, \ldots, Q_{m}\right)$ is the geometric realization of $Q_{1} * \cdots * Q_{m}$, their flag- $\ell$ polynomials are identical, and we have the following corollary.

Corollary 4.4 For $\ell \in \mathbb{N}$ and simplicially embedded polytopes $Q_{1}, \ldots, Q_{m}$, the flag- $\ell$ polynomial for the $m$-fold free join $Q_{1} * \cdots * Q_{m}$ is given by

$$
\tilde{f}_{Q_{1} * \cdots * Q_{m}}^{\ell}\left(x_{1}, \ldots, x_{\ell}\right)=\sum_{\tilde{A} \in \mathbf{C}_{\ell}\left(\Delta_{m-1}\right)}\left(\tilde{x}^{\partial(\operatorname{dim}(\tilde{A}))} \prod_{i=1}^{\ell}\left(\prod_{\alpha_{i} \in \operatorname{supp}\left(A_{i}\right) \backslash \operatorname{supp}\left(A_{i-1}\right)} \tilde{f}_{\left.Q_{\alpha_{i}}^{\ell-i+1}\left(x_{i}, \ldots, x_{\ell}\right)\right)}\right) .\right.
$$

We now consider a generalization $P\left(Q_{1}, \ldots, Q_{m}\right)$ of $\Delta_{m-1}\left(Q_{1}, \ldots, Q_{m}\right)$ where $P$ is now an arbitrary geometric polytope of dimension $m-1$ with $\operatorname{supp}(P)=\{1, \ldots, m\}$. Here we assume $P=\operatorname{conv}\left(\tilde{p}_{1}, \ldots, \tilde{p}_{n}\right)$ to be simplicially embedded in $\mathbb{R}^{m}$ and $\tilde{p}_{i}=\left(p_{i 1}, \ldots, p_{i m}\right)$ for each $i$. The polytope $P$ acts on simplicially embedded $Q_{1}, \ldots, Q_{m}$ as $\left(Q_{1}, \ldots, Q_{m}\right) \mapsto P\left(Q_{1}, \ldots, Q_{m}\right)$ and the action is given by the following.

Definition 4.5 For a simplicially embedded polytope $P=\operatorname{conv}\left(\tilde{p}_{1}, \ldots, \tilde{p}_{n}\right)$ with $\operatorname{supp}(P)=\{1, \ldots, m\}$ and simplicially embedded polytopes $Q_{1}, \ldots, Q_{m}$ the action of $P$ is given by

$$
\begin{aligned}
P\left(Q_{1} \ldots, Q_{m}\right):= & \left\{t_{1}\left(p_{11} \tilde{x}_{1}, \ldots, p_{1 m} \tilde{x}_{m}\right)+\cdots+t_{n}\left(p_{n 1} \tilde{x}_{1}, \ldots, p_{n m} \tilde{x}_{m}\right):\right. \\
& \left.0 \leq t_{i} \leq 1, \sum_{i} t_{i}=1, \tilde{x}_{i} \in Q_{i} \text { for each } i\right\} .
\end{aligned}
$$

Similar to Observation 4.3 we have the following generalization.

Proposition 4.6 The dimension of the polytope $P\left(Q_{1}, \ldots, Q_{m}\right)$ from Definition 4.5 is given by $m-1+\sum_{i=1}^{m} \operatorname{dim}\left(Q_{i}\right)$ and it is simplicially embedded. Each full face has the form $A\left(F_{1}, \ldots, F_{m}\right)$ where $A$ is a full face of $P$ and each $F_{i}$ is a full face of $Q_{i}$. Also

$$
\operatorname{dim}\left(A\left(F_{1}, \ldots, F_{m}\right)\right)=\operatorname{dim}(A)+\sum_{i \in \operatorname{supp}(A)} \operatorname{dim}\left(F_{i}\right) .
$$

Proof. For each $i$ let $d_{i}=\operatorname{dim}\left(Q_{i}\right)$ and $\tilde{x}_{i} \in \mathbb{R}^{d_{i}+1}$ a tuple of variables and $\tilde{x}^{*}=\left(\tilde{x}_{1}, \ldots, \tilde{x}_{m}\right) \in$ $\mathbb{R}^{m+\sum_{i} d_{i}}$. Each full face of $P\left(Q_{1} \ldots, Q_{m}\right)$ is determined as the maximum of a functional $\tilde{x}^{*} \mapsto \tilde{a}^{*} \cdot \tilde{x}^{*}$. For a given $\tilde{a}^{*}=\left(\tilde{a}_{1}, \ldots, \tilde{a}_{m}\right)$ and $i$, let $F_{i}$ be the face of $Q_{i}$ that maximizes $\tilde{x}_{i} \mapsto \tilde{a}_{i} \cdot \tilde{x}_{i}$ and assume this maximum is $M_{i}$. Let $A$ be the face of $P$ that is maximizes $\left(y_{1}, \ldots, y_{m}\right) \mapsto \sum_{i} M_{i} y_{i}$. Then the face that maximizes $\tilde{x}^{*} \mapsto \tilde{a}^{*} \cdot \tilde{x}^{*}$ is $A\left(F_{1}, \ldots, F_{m}\right)$.

REmark: Note that if $i \notin \operatorname{supp}(A)$ then $A\left(F_{1}, \ldots, F_{m}\right)=A\left(F_{1}, \ldots, F_{i-1}, Q_{i}, F_{i+1}, \ldots, F_{m}\right)$ regardless of the face $F_{i}$.

We now briefly discuss how we interpret $P\left(Q_{1} \ldots, Q_{m}\right)$ when $Q_{i}=\emptyset$ is allowed: let $i \in$ $\{1, \ldots, m\}$ and assume that $Q_{i}=\emptyset$ is the empty polytope. As $P=\operatorname{conv}\left(\tilde{p}_{1}, \ldots, \tilde{p}_{n}\right)$ is simplicially embedded in $\mathbb{R}^{m}$, we can assume that $i \notin \operatorname{supp}\left(\tilde{p}_{1}, \ldots, \tilde{p}_{h}\right)$ and $i \in \operatorname{supp}\left(\tilde{p}_{h+1}, \ldots, \tilde{p}_{n}\right)$. In this case let $P_{\hat{\imath}}=\operatorname{conv}\left(\pi_{\hat{\imath}}\left(\tilde{p}_{1}\right), \ldots, \pi_{\hat{\imath}}\left(\tilde{p}_{h}\right)\right)$ where $\pi_{\hat{\imath}}: \mathbb{R}^{m} \rightarrow \mathbb{R}^{m-1}$ is the projection obtained by omitting the $i$-th coordinate. In this case we make the following definition.

$$
P\left(Q_{1} \ldots, Q_{m}\right)=P\left(Q_{1} \ldots, Q_{i-1}, \emptyset, Q_{i+1}, \ldots, Q_{m}\right):=P_{\hat{\imath}}\left(Q_{1}, \ldots, Q_{i-1}, Q_{i+1}, \ldots, Q_{m}\right) .
$$


By (5) we can therefore assume that each $Q_{i}$ in $P\left(Q_{1} \ldots, Q_{m}\right)$ is a proper nonempty polytope.

We now state and prove our main result in this section, that the $\ell$-flag polynomial for $P\left(Q_{1}, \ldots, Q_{m}\right)$ has the same form as displayed in Corollary 4.4.

Theorem 4.7 For $\ell \in \mathbb{N}$ and simplicially embedded polytopes $Q_{1}, \ldots, Q_{m}$, the flag- $\ell$ polynomial of the polytope $P\left(Q_{1}, \ldots, Q_{m}\right)$ from Definition 4.5 is given by

$$
\tilde{f}_{P\left(Q_{1}, \ldots, Q_{m}\right)}^{\ell}\left(x_{1}, \ldots, x_{\ell}\right)=\sum_{\tilde{A} \in \mathbf{C}_{\ell}(P)}\left(\tilde { x } ^ { \partial ( \operatorname { d i m } ( \tilde { A } ) ) } \prod _ { i = 1 } ^ { \ell } \left(\prod_{\alpha_{i} \in \operatorname{supp}\left(A_{i}\right) \backslash \operatorname{supp}\left(A_{i-1}\right)} \tilde{f}_{\left.\left.Q_{\alpha_{i}}^{\ell-i+1}\left(x_{i}, \ldots, x_{\ell}\right)\right)\right) .}\right.\right.
$$

We will prove Theorem 4.7 in a few steps and use that to prove Theorem 3.3. For simplicity we will write $\mathbf{X}=\left(X_{1}, \ldots, X_{m}\right)$. Assuming that $\operatorname{dim}\left(Q_{i}\right)=d_{i}$ for each $i$ we have linear maps $h=h_{P(\mathbf{Q})}: \mathbb{R}^{m+\sum d_{i}} \rightarrow \mathbb{R}^{m}$ and $\pi_{\tilde{\imath}}: \mathbb{R}^{m+\sum d_{i}} \rightarrow \mathbb{R}^{d_{i}+1}$ given by

$$
h\left(\tilde{x}_{1}, \ldots, \tilde{x}_{m}\right)=\left(\sum_{i=1}^{d_{1}} x_{1 i}, \ldots, \sum_{i=1}^{d_{m}} x_{m i}\right)
$$

and

$$
\pi_{\tilde{\imath}}\left(\tilde{x}_{1}, \ldots, \tilde{x}_{m}\right)=\tilde{x}_{i}
$$

respectively.

Lemma 4.8 For faces of the polytope $P(\mathbf{Q})$ we have $A(\mathbf{F}) \subseteq A^{\prime}\left(\mathbf{F}^{\prime}\right)$ if and only if $A \subseteq A^{\prime}$ are subfaces in $P$ and $F_{i} \subseteq F_{i}^{\prime}$ are subfaces in $Q_{i}$ for each $i \in \operatorname{supp}(A)$.

Proof. We will prove the more involved direction. Assume that $A(\mathbf{F}) \subseteq A^{\prime}\left(\mathbf{F}^{\prime}\right)$. Since each of the $Q_{i}$ are simplicially embedded, we have $A=h(A(\mathbf{F})) \subseteq h\left(A^{\prime}\left(\mathbf{F}^{\prime}\right)\right)=A^{\prime}$.

For $i \in \operatorname{supp}(A)$, then by the definition of $P(\mathbf{Q})=P\left(Q_{1}, \ldots, Q_{m}\right)$, we have $\pi_{\tilde{\imath}}(A(\mathbf{F}))=\left[a_{i}, b_{i}\right] F_{i}$ where $\pi_{i}(A)=\left[a_{i}, b_{i}\right]$ and $0 \leq a_{i}<b_{i} \leq 1$. (Here $\pi_{i}$ is the projection onto the $i$-th coordinate). Since we have that $A \subseteq A^{\prime}$, then for each $i$ we have $\left[a_{i}, b_{i}\right] \subseteq\left[a_{i}^{\prime}, b_{i}^{\prime}\right]$ or $a_{i}^{\prime} \leq a_{i}<b_{i} \leq b_{i}^{\prime}$, or

$$
\left[a_{i}, b_{i}\right] F_{i}=\pi_{\tilde{\imath}}(A(\mathbf{F})) \subseteq \pi_{\tilde{\imath}}\left(A^{\prime}\left(\mathbf{F}^{\prime}\right)\right)=\left[a_{i}^{\prime}, b_{i}^{\prime}\right] F_{i}^{\prime} .
$$

For $c_{i} \in\left[a_{i}, b_{i}\right]$ we have $c_{i} F_{i} \subseteq\left[a_{i}, b_{i}\right] F_{i} \subseteq\left[a_{i}^{\prime}, b_{i}^{\prime}\right] F_{i}^{\prime}$. Since $c_{i} F_{i}=\left\{\tilde{x} \in\left[a_{i}, b_{i}\right] F_{i}: \sum_{j} x_{j}=c_{i}\right\}$, then $c_{i} F_{i} \subseteq\left[a_{i}^{\prime}, b_{i}^{\prime}\right] F_{i}^{\prime} \cap\left\{\tilde{x}: \sum_{j} x_{j}=c_{i}\right\}=c_{i} F_{i}^{\prime}$ (Here $Q_{i}$ is simplicially embedded and so are all of its faces). Therefore

$$
F_{i}=\frac{1}{c_{i}}\left(c_{i} F_{i}\right) \subseteq \frac{1}{c_{i}}\left(c_{i} F_{i}^{\prime}\right)=F_{i}^{\prime}
$$

which completes the proof of the lemma.

By Proposition 4.6 each $\ell$-chain of faces of $P(\mathbf{Q})$ has the form

$$
A_{1}\left(\mathbf{F}_{1}\right) \subseteq A_{2}\left(\mathbf{F}_{2}\right) \subseteq \cdots \subseteq A_{\ell}\left(\mathbf{F}_{\ell}\right)
$$

which by Lemma 4.8 firstly yields a unique $\ell$-chain $\tilde{A}: A_{1} \subseteq A_{2} \subseteq \cdots \subseteq A_{\ell}$ of faces of $P$, and hence $\operatorname{supp}\left(A_{1}\right) \subseteq \operatorname{supp}\left(A_{2}\right) \subseteq \cdots \subseteq \operatorname{supp}\left(A_{\ell}\right)$. By a suitable permutation of the $\{1, \ldots, m\}$ we may assume that $\operatorname{supp}\left(A_{1}\right)<\operatorname{supp}\left(A_{2}\right) \backslash \operatorname{supp}\left(A_{1}\right)<\cdots<\operatorname{supp}\left(A_{\ell}\right) \backslash \operatorname{supp}\left(A_{\ell-1}\right)$ (where $X<Y$ 
means that $x<y$ for all $x \in X$ and $y \in Y)$. Secondly, by this mentioned assumption on the order on the $\operatorname{supp}\left(A_{i}\right)$, then for each fixed $\ell$-chain $\tilde{A} \in \mathbf{C}_{\ell}(P)$ (6) yields a Young tableau (or Ferres diagram) of boxes in $[\ell] \times[m]$ such that for each $i \in[\ell]$ the boxes numbered $(i, 1), \ldots,\left(i, a_{i}\right)$, where $a_{i}=\left|\operatorname{supp}\left(A_{i}\right)\right|$, contain the faces $F_{i 1}, F_{i 2}, \ldots, F_{i a_{i}}$ from $\mathbf{F}_{i}$ that are indexed by numbers of $\operatorname{supp}\left(A_{i}\right)$. Let $1=i_{1}<i_{2}<\cdots<i_{\lambda} \leq \ell$ be the complete list of numbers from $[\ell]$ where $a_{i_{j}-1}<a_{i_{j}}$ for each $j=1, \ldots, \lambda$. Hence, for each such $j$ we obtain $a_{i_{j}}-a_{i_{j}-1}$ chains of faces

$$
F_{i_{j} \alpha} \subseteq F_{i_{j}+1 \alpha} \subseteq \cdots \subseteq F_{\ell \alpha},
$$

in $Q_{\alpha}$, one for each $\alpha \in \operatorname{supp}\left(A_{i_{j}}\right) \backslash \operatorname{supp}\left(A_{i_{j}-1}\right)$. Conversely, every $\ell$-chain as in (6) is presented by such a Young tableau in which the horizontal sequence of boxes at hight $\alpha$ yields a chain of faces in $Q_{\alpha}$. This together with Proposition 4.6 yields the following lemma.

Lemma 4.9 For $\ell \in \mathbb{N}$ let $\tilde{A} \in \mathbf{C}_{\ell}(P)$ be a fixed $\ell$-chain of faces of $P$. Then the coefficient of $\tilde{x}^{\partial \tilde{s}}$ in the polynomial given by

$$
\tilde{x}^{\partial(\operatorname{dim}(\tilde{A}))} \prod_{j=1}^{\lambda}\left(\prod_{\alpha_{j} \in \operatorname{supp}\left(A_{i_{j}}\right) \backslash \operatorname{supp}\left(A_{i_{j-1}}\right)} \tilde{f}_{Q_{\alpha_{j}}}^{\ell-i_{j}+1}\left(x_{i_{j}}, \ldots, x_{\ell}\right)\right)
$$

is the number of $\ell$-chains $C_{1} \subseteq \cdots \subseteq C_{\ell}$ of faces of $P\left(Q_{1}, \ldots, Q_{m}\right)$ with $\operatorname{dim}\left(C_{i}\right)=s_{i}$ and $h\left(C_{i}\right)=$ $A_{i}$.

With the convention that a product with an empty index set equals one, (77) can be written as

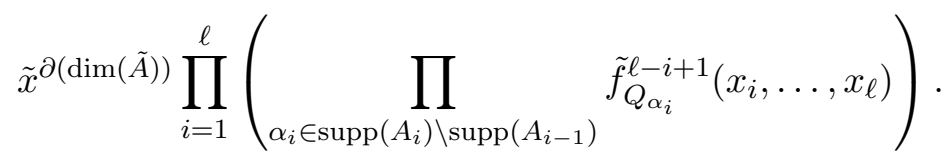

Theorem 4.7 now follows directly from Lemma 4.9 .

Now let $k, r \in \mathbb{N}$ and consider a Minkowski sum $P_{\mathcal{F}}$ of simplices as in (11). For $i \in\left[2^{k}-1\right]$ let $r_{i}$ be as before, the number of elements of $R_{i}=\left\{j \in[r]: N_{\mathcal{F}}(j)=N_{\mathcal{H}(k)}(i)\right\}$ and so $r=r_{1}+\cdots+r_{2^{k}-1}$. If $n \in \mathbb{N}$ and $C$ is a convex set, then $C+\cdots+C$ ( $n$ times $)=n C$. Hence, from this and by Definitions 3.1 of the master polytope $P(k)$ and 4.5 we have that

$$
\begin{aligned}
P_{\mathcal{F}} & =\sum_{F \in \mathcal{F}} \Delta_{F} \\
& =\Delta_{F_{1}}+\cdots+\Delta_{F_{k}} \\
& =P(k)\left(\Delta_{\left[r_{1}\right]}, \Delta_{\left[r_{2}\right]}, \ldots, \Delta_{\left[r_{2^{k}-1}\right]}\right) \\
& =P(k)\left(\Delta_{r_{1}-1}, \Delta_{r_{2}-1}, \ldots, \Delta_{r_{2^{k}-1}-1}\right) .
\end{aligned}
$$

Theorem 3.3 now follows from (8) and Theorem 4.7.

\section{Extremal considerations of the Minkowski sum of two simplices}

In this section we consider two extremal examples of the Minkowski sum of two simplices, where we apply the flag polynomial. In our first example we obtain the $f$-polynomial of a general Minkowski 
sum of $k$ standard simplices in terms of the master polytope and use that to compute the maximum number of $d$-faces of the Minkowski sum of two simplices when $k=2$. In our second example we compute the maximum discrepancy between the number of $(0, d)$-chains of faces of the Minkowski sum and the minimum number of such $(0, d)$-chains of a simple polytope of the same dimension and on the same number of vertices.

\subsection{First Example}

As before, $P_{\mathcal{F}}$ will be the Minkowski sum of $k$ simplices as in (1). Note that each face $Q$ of $P_{\mathcal{F}}$ can be viewed as a 1 -chain of faces. Hence, letting $\ell=1$ in Theorem 3.3 we get $\mathbf{C}_{1}(P(k))=\mathbf{f}(P(k))$, the set of faces of $P(k)$, and we obtain a generating function in terms of $x_{1}$ alone, which we denote by just $x$ in the following corollary.

Corollary 5.1 For a collection of $k$ ordered subsets $\mathcal{F}=\left(F_{1}, F_{2}, \ldots, F_{k}\right)$ of $[r]$, the $f$-polynomial of the Minkowski sum $P_{\mathcal{F}}$ is given by

$$
f_{P_{\mathcal{F}}}(x)=\sum_{A \in \mathbf{f}(P(k))}\left(x^{\operatorname{dim}(A)} \prod_{i \in \operatorname{supp}(A)} f_{\Delta_{r_{i}-1}}(x)\right) .
$$

We will now use Corollary 5.1 to investigate in more detail the Minkowski sum of two simplices, the $f$-polynomial and then the maximum number of $d$-dimensional faces such a polytope can have.

By Corollary 5.1 we have in particular for $k=2$ and $\mathcal{F}=\left(F_{1}, F_{2}\right)$ (and therefore $r_{1}=\left|F_{1} \cap F_{2}\right|$, $r_{2}=\left|F_{1} \backslash F_{2}\right|$ and $\left.r_{3}=\left|F_{2} \backslash F_{1}\right|,\right)$ that the $f$-polynomial of $P_{\mathcal{F}}=\Delta_{F_{1}}+\Delta_{F_{2}}$ is given by

$$
\begin{aligned}
f_{P_{\mathcal{F}}}(x) & =\left(x^{2}+2 x\right) f_{\Delta_{r_{1}-1}}(x) f_{\Delta_{r_{2}-1}}(x) f_{\Delta_{r_{3}-1}}(x) \\
& +(x+1)\left(f_{\Delta_{r_{1}-1}}(x) f_{\Delta_{r_{2}-1}}(x)+f_{\Delta_{r_{1}-1}}(x) f_{\Delta_{r_{3}-1}}(x)\right) \\
& +f_{\Delta_{r_{2}-1}}(x) f_{\Delta_{r_{3}-1}}(x) \\
& +f_{\Delta_{r_{1}-1}}(x) .
\end{aligned}
$$

Hence, together with Observation 1.1 we have the following.

Corollary 5.2 If $P_{\mathcal{F}}=\Delta_{F_{1}}+\Delta_{F_{2}}$ and $r=r_{1}+r_{2}+r_{3}$, where $r_{1}=\left|F_{1} \cap F_{2}\right|, r_{2}=\left|F_{1} \backslash F_{2}\right|$ and $r_{3}=\left|F_{2} \backslash F_{1}\right|$, then the $f$-polynomial of $P_{\mathcal{F}}$ is given by

$$
f_{P_{\mathcal{F}}}(x)=\frac{1}{x^{2}}\left((x+2)(x+1)^{r}-(x+1)^{r_{1}+r_{2}}-(x+1)^{r_{1}+r_{3}}-(x+1)^{r_{2}+r_{3}+1}+1\right),
$$

and hence for each $d \in\{0,1, \ldots, r-1\}$ the number $f_{d}\left(P_{\mathcal{F}}\right)$ of $d$-dimensional faces of $P_{\mathcal{F}}$ is given by

$$
f_{d}\left(P_{\mathcal{F}}\right)=\left(\begin{array}{c}
r+1 \\
d+2
\end{array}\right)+\left(\begin{array}{c}
r \\
d+2
\end{array}\right)-\left(\begin{array}{c}
r_{1}+r_{2} \\
d+2
\end{array}\right)-\left(\begin{array}{c}
r_{1}+r_{3} \\
d+2
\end{array}\right)-\left(\begin{array}{c}
r_{2}+r_{3}+1 \\
d+2
\end{array}\right) .
$$

REMARK: Writing $f_{P_{\mathcal{F}}}(x)$ directly in terms of sets $F_{1}, F_{2}, F_{3}$ we obtain

$$
f_{P_{\mathcal{F}}}(x)=\frac{1}{x^{2}}\left((x+2)(x+1)^{\left|F_{1} \cup F_{2}\right|}-(x+1)^{\left|F_{1}\right|}-(x+1)^{\left|F_{2}\right|}-(x+1)^{\left|F_{1} \triangle F_{2}\right|+1}+1\right) .
$$


For given $r \in \mathbb{N}$ and $d \in\{0,1, \ldots, r-1\}$ we want to find the the maximum number $f_{d}(r)$ of $d$-dimensional faces a Minkowski sum $P_{\mathcal{F}}=\Delta_{F_{1}}+\Delta_{F_{2}}$ of two simplices with $F_{1}, F_{2} \subseteq\{1, \ldots, r\}$ can have. That is, we want to determine

$$
f_{2 ; d}(r)=\max \left\{f_{d}\left(P_{\mathcal{F}}\right): P_{\mathcal{F}}=\Delta_{F_{1}}+\Delta_{F_{2}}, \quad r_{1}+r_{2}+r_{3}=r\right\} .
$$

To do this, it is understood that for a nonzero integer $c$ the map $\mathbb{Z} \rightarrow \mathbb{Z}$ defined as $x \mapsto\left(\begin{array}{l}x \\ c\end{array}\right)$ is given by $\left(\begin{array}{l}x \\ c\end{array}\right)=\frac{x(x-1) \cdots(x-c+1)}{c !}$ for $x \geq c$ and $\left(\begin{array}{l}x \\ c\end{array}\right)=0$ for $x<c$.

Lemma 5.3 For nonzero integers $c$ and $x<y$ we have that

$$
\left(\begin{array}{l}
x \\
c
\end{array}\right)+\left(\begin{array}{l}
y \\
c
\end{array}\right) \geq\left(\begin{array}{c}
x+1 \\
c
\end{array}\right)+\left(\begin{array}{c}
y-1 \\
c
\end{array}\right)
$$

Proof. Considering each of the cases, $c \leq x<y, x<c<y$ and $x<y \leq c$, and using the fact that $\left(\begin{array}{c}t+1 \\ c\end{array}\right)-\left(\begin{array}{c}t \\ c\end{array}\right)=\left(\begin{array}{c}t \\ c-1\end{array}\right)$ when $t \geq c-1$, the lemma follows.

Corollary 5.4 Let $K \in \mathbb{N}$ and $x_{1}, x_{2}, x_{3} \geq 0$ be integers with $x_{1}+x_{2}+x_{3}=K$ and

$$
g\left(x_{1}, x_{2}, x_{3}\right)=\left(\begin{array}{c}
x_{1} \\
c
\end{array}\right)+\left(\begin{array}{c}
x_{2} \\
c
\end{array}\right)+\left(\begin{array}{c}
x_{3} \\
c
\end{array}\right) .
$$

- If $K \geq 3 c-2$, then $g\left(x_{1}, x_{2}, x_{3}\right)$ will take it minimum value if, and only if, $\left|x_{i}-x_{j}\right| \leq 1$ for each $i, j$.

- If $K \leq 3(c-1)$, then any $x_{1}, x_{2}, x_{3} \leq c-1$ summing up to $K$ will yield the minimum value 0 of $g\left(x_{1}, x_{2}, x_{3}\right)$. In particular those with $\left|x_{i}-x_{j}\right| \leq 1$ for each $i, j$.

Clearly $f_{d}\left(P_{\mathcal{F}}\right)$ from Corollary 5.2 is maximized iff

$$
h\left(r_{1}, r_{2}, r_{3}\right)=\left(\begin{array}{c}
r_{1}+r_{2} \\
d+2
\end{array}\right)+\left(\begin{array}{c}
r_{1}+r_{3} \\
d+2
\end{array}\right)+\left(\begin{array}{c}
r_{2}+r_{3}+1 \\
d+2
\end{array}\right) .
$$

is minimized. Note that $\left(r_{1}+r_{2}\right)+\left(r_{1}+r_{3}\right)+\left(r_{2}+r_{3}+1\right)=2 r+1$. Considering each of the cases of $r \equiv 0,1,2(\bmod 3)$ we obtain the following theorem, in which $[x]$ denotes the nearest integer to the real number $x$. Note that for a fixed $x=a / b$, where $a, b$ are integers and $b$ is odd, then $y=[x]$ is the unique integer that minimizes $|x-y|$.

Theorem 5.5 If $f_{2 ; d}(r)$ is the maximum number of d-faces in a Minkowski sum $\Delta_{F_{1}}+\Delta_{F_{2}}$ of two simplices where $F_{1}, F_{2} \subseteq\{1, \ldots, r\}$ as given in (9), then

$$
f_{2 ; d}(r)=\left(\begin{array}{c}
r+1 \\
d+2
\end{array}\right)+\left(\begin{array}{c}
r \\
d+2
\end{array}\right)-\left(\begin{array}{c}
\lfloor(2 r+1) / 3\rfloor \\
d+2
\end{array}\right)-\left(\begin{array}{c}
{[(2 r+1) / 3]} \\
d+2
\end{array}\right)-\left(\begin{array}{c}
\lceil(2 r+1) / 3\rceil \\
d+2
\end{array}\right) .
$$

REMARKS: (i) In the case when $2(r-1) / 3 \leq d \leq r-1$ we note that $f_{2 ; d}(r)=\left(\begin{array}{c}r+1 \\ d+2\end{array}\right)+\left(\begin{array}{c}r \\ d+2\end{array}\right)$. Moreover, any Minkowski sum $P_{\mathcal{F}}=\Delta_{F_{1}}+\Delta_{F_{2}}$ such that $r_{1}+r_{2}+r_{3}=r, r_{1} \geq r-d$ and $r_{2}, r_{3} \geq r-d+1$ will have $f_{d}\left(P_{\mathcal{F}}\right)=f_{2 ; d}(r)$ in this case. (ii) Otherwise, if $0 \leq d<2(r-1) / 3$, then the Minkowski sum $P_{\mathcal{F}}=\Delta_{F_{1}}+\Delta_{F_{2}}$ with $f_{d}\left(P_{\mathcal{F}}\right)=f_{2 ; d}(r)$ is more "unique" in the following sense: 
If $r=3 q$, then $F_{1}$ and $F_{2}$ must satisfy

$$
\left(r_{1}, r_{2}, r_{3}\right) \in\{(q, q, q),(q+1, q, q-1),(q+1, q-1, q)\} .
$$

If $r=3 q+1$, then $F_{1}$ and $F_{2}$ must satisfy $\left(r_{1}, r_{2}, r_{3}\right)=(q+1, q, q)$.

If $r=3 q+2$, then $F_{1}$ and $F_{2}$ must satisfy

$$
\left(r_{1}, r_{2}, r_{3}\right) \in\{(q+2, q, q),(q+1, q+1, q),(q+1, q, q+1)\} .
$$

Directly from Theorem 5.5 we have the following.

Corollary 5.6 For a fixed $d$ we have

$$
\lim _{r \rightarrow \infty} \frac{f_{2 ; d}(r)}{r^{d+2}}=\frac{2\left(3^{d+1}-2^{d+1}\right)}{3^{d+1}(d+2) !}
$$

\subsection{Second Example}

Consider the Minkowski sum $P_{\mathcal{F}}=\Delta_{F_{1}}+\Delta_{F_{2}}$ of two sets $\mathcal{F}=\left(F_{1}, F_{2}\right)$, where $r=r_{1}+r_{2}+r_{3}$, $r_{1}=\left|F_{1} \cap F_{2}\right|, r_{2}=\left|F_{1} \backslash F_{2}\right|$ and $r_{3}=\left|F_{2} \backslash F_{1}\right|$ as before. Directly by Corollary 5.2 we have that $f_{0}:=f_{0}\left(P_{\mathcal{F}}\right)$, the number of vertices of $P_{\mathcal{F}}$, is given by $f_{0}=r_{1} r_{2}+r_{1} r_{3}+r_{2} r_{3}+r_{1}$, something which we saw alternatively in the last example of Section 3. If $F_{1}$ and $F_{2}$ are disjoint, then $P_{\mathcal{F}}=\Delta_{F_{1}} \times \Delta_{F_{2}}$ (see Proposition 1.2 in [8] for a more general result) which is a simple polytope by the simplicity of any simplex. Hence we will assume that $F_{1}$ and $F_{2}$ have a nonempty intersection so $r_{1} \geq 1$, in which case the dimension of $P_{\mathcal{F}}$ is precisely $r-1$ (see [8] for more detailed discussion).

For a polynomial $f(\tilde{x})$, denote by $\left[\tilde{x}^{\tilde{a}}\right] f(\tilde{x})$ the coefficient for $\tilde{x}^{\tilde{a}}$ in $f(\tilde{x})$. Note that the number of $(0, d)$-chains in $P_{\mathcal{F}}=\Delta_{F_{1}}+\Delta_{F_{2}}$ is then given by $\left[x_{2}^{d}\right] \tilde{f}_{P_{\mathcal{F}}}^{2}\left(x_{1}, x_{2}\right)$. Note that when $F_{1}$ and $F_{2}$ are not disjoint, then each vertex of $P_{\mathcal{F}}$ is contained in a minimum of $\left(\begin{array}{c}r-1 \\ d\end{array}\right) d$-faces since the degree of each vertex is at least $r-1$ and $d$ of these yield a face.

Observation 5.7 For $P_{\mathcal{F}}=\Delta_{F_{1}}+\Delta_{F_{2}}$ where $F_{1}$ and $F_{2}$ have a nonempty intersection, $P_{\mathcal{F}}$ is simple iff

$$
\left[x_{2}^{d}\right] \tilde{f}_{P_{\mathcal{F}}}^{2}\left(x_{1}, x_{2}\right)=\left(\begin{array}{c}
r-1 \\
d
\end{array}\right) f_{0}=\left(\begin{array}{c}
r-1 \\
d
\end{array}\right)\left(r_{1} r_{2}+r_{1} r_{3}+r_{2} r_{3}+r_{1}\right) .
$$

Since $\left[x_{2}^{d}\right] \tilde{f}_{P_{\mathcal{F}}}^{2}\left(x_{1}, x_{2}\right)=\left[x_{2}^{d}\right] \tilde{f}_{P_{\mathcal{F}}}^{2}\left(0, x_{2}\right)$, and in addition $\tilde{f}_{\Delta_{r_{i}-1}}^{2}(0, x)=r_{i}(x+1)^{r_{i}-1}$ and $\tilde{f}_{\Delta_{r_{i}-1}}^{1}(x)=$ $\left((x+1)^{r_{i}}-1\right) / x$, we obtain by Corollary 3.4 that

$$
\begin{aligned}
f_{(0, d)}\left(P_{\mathcal{F}}\right) & =\left[x^{d}\right] \tilde{f}_{P_{\mathcal{F}}}^{2}(0, x) \\
& =\left[x^{d}\right]\left(\left(r_{1} r_{2}+r_{1} r_{3}+r_{2} r_{3}+r_{1}\right)(x+1)^{r-1}+r_{2} r_{3}(x+1)^{r-2}-r_{2} r_{3}(x+1)^{r_{2}+r_{3}-1}\right),
\end{aligned}
$$

and hence we have the following.

Corollary 5.8 The number $f_{(0, d)}\left(P_{\mathcal{F}}\right)$ of $(0, d)$-chains in $P_{\mathcal{F}}=\Delta_{F_{1}}+\Delta_{F_{2}}$ is given by

$$
f_{(0, d)}\left(P_{\mathcal{F}}\right)=\left(r_{1} r_{2}+r_{1} r_{3}+r_{2} r_{3}+r_{1}\right)\left(\begin{array}{c}
r-1 \\
d
\end{array}\right)+r_{2} r_{3}\left(\begin{array}{c}
r-2 \\
d
\end{array}\right)-r_{2} r_{3}\left(\begin{array}{c}
r_{2}+r_{3}-1 \\
d
\end{array}\right) .
$$


By Observation 5.7 and Corollary 5.8 it is immediate that the Minkowski sum $P_{\mathcal{F}}=\Delta_{F_{1}}+\Delta_{F_{2}}$ is simple iff $r_{2}=0, r_{3}=0$ or $r_{1}=1$.

The other question "when is $P_{\mathcal{F}}=\Delta_{F_{1}}+\Delta_{F_{2}}$ farthest from being simple?" is more interesting. To make this question precise and address it properly, we define the discrepancy $\delta_{(0, d)}\left(P_{\mathcal{F}}\right)$ as the difference between $f_{(0, d)}\left(P_{\mathcal{F}}\right)$ and the minimum number $\left(\begin{array}{c}r-1 \\ d\end{array}\right) f_{0}$ of $(0, d)$-chains in simple polytope of the same dimension and on the same number $f_{0}=r_{1} r_{2}+r_{1} r_{3}+r_{2} r_{3}+r_{1}$ of vertices,

$$
\delta_{(0, d)}\left(P_{\mathcal{F}}\right):=f_{(0, d)}\left(P_{\mathcal{F}}\right)-\left(\begin{array}{c}
r-1 \\
d
\end{array}\right) f_{0}=r_{2} r_{3}\left(\left(\begin{array}{c}
r-2 \\
d
\end{array}\right)-\left(\begin{array}{c}
r_{2}+r_{3}-1 \\
d
\end{array}\right)\right) .
$$

The discrepancy $\delta_{(0, d)}\left(P_{\mathcal{F}}\right)$ can be thought of as a measure (in terms of $d$ ) on how far from simple the polytope $P_{\mathcal{F}}$ is. Hence, for a fixed $r \in \mathbb{N}$ we like to determine what kind of Minkowski sum $P_{\mathcal{F}}$ of two simplices will yield the largest discrepancy. This is only of interest if $r_{2} r_{3} \neq 0$ and $r_{1} \geq 1$,

$$
\delta_{2 ;(0, d)}(r):=\max \left\{\delta_{(0, d)}\left(P_{\mathcal{F}}\right): P_{\mathcal{F}}=\Delta_{F_{1}}+\Delta_{F_{2}}, \quad r_{1}+r_{2}+r_{3}=r, \quad r_{1} \geq 1\right\} .
$$

Since $r_{1} \geq 1$ then $r_{2}+r_{3} \leq r-1$, so to determine $\delta_{2 ;(0, d)}(r)$ it suffices to compute $\max (f)$ where $f(x, y)$ is given by

$$
f(x, y)= \begin{cases}x y\left(\begin{array}{c}
r-2 \\
d
\end{array}\right) & \text { if } 0 \leq x+y \leq d, \quad x, y \geq 0 \\
x y\left(\left(\begin{array}{c}
r-2 \\
d
\end{array}\right)-\left(\begin{array}{c}
x+y-1 \\
d
\end{array}\right)\right) & \text { if } d+1 \leq x+y \leq r-1, \quad x, y \geq 0 .\end{cases}
$$

Considering $f(x, y)$ in integers $x$ and $y$ on each line $x+y=c$ where $c$ is a constant, then clearly the maximum of $f(x, y)$ is reached on each of these lines when $|x-y| \in\{0,1\}$. In particular, when $0 \leq x+y \leq d$ the maximum of $f(x, y)$ is $\lfloor d / 2\rfloor\lceil d / 2\rceil\left(\begin{array}{c}r-2 \\ d\end{array}\right)$. For the other range $d+1 \leq x+y \leq r-1$, the more interesting one (since we like to consider $d$ fixed and $r$ much larger than $d$ ), we can by symmetry assume that $x \leq y$ and write $y=x+\epsilon$ where $\epsilon \in\{0,1\}$. Then by expressing $f(x, y)=f(x, x+\epsilon)$ in terms of $z=x+y-1=2 x+\epsilon-1$, we have that $\delta_{2 ;(0, d)}(r)=\max (g)$ where $g(z)=\frac{1}{4}(z+1-\epsilon)(z+1+\epsilon)\left(\left(\begin{array}{c}r-2 \\ d\end{array}\right)-\left(\begin{array}{c}z \\ d\end{array}\right)\right)$ and $d \leq z \leq r-2$. For the moment we consider real $z$ in the mentioned interval, so by the intermediate value theorem for real continuous functions, $\max (g)$ is obtained at $g\left(z^{*}\right)$ where $z-1 \leq z^{*} \leq z$ and the difference $\Delta g(z-1)=g(z)-g(z-1)=0$, or

$$
(z+1-\epsilon)(z+1+\epsilon)\left(\left(\begin{array}{c}
r-2 \\
d
\end{array}\right)-\left(\begin{array}{l}
z \\
d
\end{array}\right)\right)=(z-\epsilon)(z+\epsilon)\left(\left(\begin{array}{c}
r-2 \\
d
\end{array}\right)-\left(\begin{array}{c}
z-1 \\
d
\end{array}\right)\right) .
$$

We will first estimate asymptotically the solution in $z$ to (11), and hence also $z^{*} \in[z-1, z]$, in terms of $d$ and $r$ and interpret the corresponding $\delta_{2 ;(0, d)}(r)=\max (g)$.

Note that (11) can be rewritten as

$$
\prod_{i=1}^{d} \frac{r-i-1}{z-i+1}=\frac{(d+2) z+1+\epsilon^{2} d / z}{2 z+1} .
$$

Considering solely the asymptotic values as $r$ tends to infinity, the solution $z$ of (12) must also tend to infinity. Hence, as the right hand side (RHS) of (12) tends to $(d+2) / 2$ as $r$ (and hence $z$ as well) goes to infinity, and the left hand side (LHS) of (12) is asymptotically $(r / z)^{d}$ as $r$ tends to infinity, we have for large $r$ that $z \sim \sqrt[d]{2 /(d+2)} r$. Hence, $\max (g)$ is obtained at $g\left(z^{*}\right)$ where $z^{*} \sim \sqrt[d]{2 /(d+2)} r$ as $r$ tends to infinity. From this we deduce the following asymptotic result. 
Proposition 5.9 If a Minkowski sum $P_{\mathcal{F}}=\Delta_{F_{1}}+\Delta_{F_{2}}$ where $F_{1}, F_{2} \subseteq\{1, \ldots, r\}$ and $r_{1} \geq 1$ has the maximum discrepancy $\delta_{2 ;(0, d)}\left(P_{\mathcal{F}}\right)=\delta_{2 ;(0, d)}(r)$ between the number of $(0, d)$-chains of faces of $P_{\mathcal{F}}$ and the minimum number $\left(\begin{array}{c}r-1 \\ d\end{array}\right) f_{0}$ of $(0, d)$-chains in a simple polytope of the same dimension and with the same number $f_{0}$ of vertices, then for all fixed $d$ and $i \in\{2,3\}$ we have

$$
\begin{aligned}
r_{1} & \sim\left(1-\sqrt[d]{\frac{2}{d+2}}\right) r \\
r_{i} & \sim \frac{1}{2} \sqrt[d]{\frac{2}{d+2}} r
\end{aligned}
$$

as $r$ tends to infinity, and hence

$$
\lim _{r \rightarrow \infty} \frac{\delta_{2 ;(0, d)}(r)}{r^{d+2}}=\frac{2^{2 / d-2}}{(d+2)^{2 / d-1}(d-1) !} .
$$

We will now determine exactly which $r_{1}, r_{2}$ and $r_{3}$ will yield the maximum discrepancy. In order to do that we determine concrete and tight upper and lower bounds for the solution $z$ of (12) to pinpoint the corresponding integer $z^{*}$ at which the maximum discrepancy $\delta_{2 ;(0, d)}(r)=$ $\max (g)=g\left(z^{*}\right)$ for the Minkowski sum $P_{\mathcal{F}}=\Delta_{F_{1}}+\Delta_{F_{2}}$ is attained. The remainder of this section will be spent computing this mentioned $z^{*}$ and interpreting the corresponding discrepancy for the Minkowski sum.

First we centralize the LHS of (12) by letting $R=r-(d+3) / 2$ and $w=z-(d-1) / 2$. In this way the LHS of (12) becomes

$$
\prod_{i=1}^{d / 2} \frac{R^{2}-(i-1 / 2)^{2}}{w^{2}-(i-1 / 2)^{2}} \quad \text { for even } d, \text { and } \quad \frac{R}{w} \prod_{i=1}^{(d-1) / 2} \frac{R^{2}-i^{2}}{y^{2}-i^{2}} \quad \text { for odd } d \text {. }
$$

Since $w<R$ are fixed then $i \mapsto h(i):=\left(R^{2}-i^{2}\right) /\left(w^{2}-i^{2}\right)$ is increasing and the RHS of (12) is at most $(d+2) / 2$, we have that $(R / w)^{d}<(d+2) / 2$ and therefore we obtain a concrete lower bound $L(d ; r)$ for $w$, and hence also for $z$, given by

$$
z>L(d ; r):=\sqrt[d]{\frac{2}{d+2}}(r-2)+\left(1-\sqrt[d]{\frac{2}{d+2}}\right) \frac{d-1}{2} .
$$

Incidentally, we will use this lower bound $L(d ; r)$ from (14) to obtain an upper bound for $z$. By (12), its LHS expressed in (13) is at least $q(z)=((d+2) z+1) /(2 z+1)$. Since $z \mapsto q(z)$ is increasing, the RHS of (12) and hence also (13) is greater than $q(L(d ; r))$. Regardless of the parity of $d$, the expression in (13) is less than $h((d-1) / 2)^{d / 2}$ and hence we obtain an upper bound for $w$, and hence also $z$, determined by $q(L(d ; r))<h((d-1) / 2)^{d / 2}$ or

$$
w<c R \sqrt{1+\left(\frac{d-1}{2}\right)^{2}\left(\frac{1}{c^{2}}-1\right) \frac{1}{R^{2}}}
$$

where $c=1 / \sqrt[d]{q(L(d ; r))}$. 
By the mean value theorem for the function $t \mapsto \sqrt[d]{t}$, there is an $\alpha \in\left[\frac{2}{d+2}, \frac{1}{q(L(d ; r))}\right]=\left[\frac{2}{d+2}, c^{d}\right]$ such that

$$
0<c-\sqrt[d]{\frac{2}{d+2}}=\frac{1}{\alpha^{1-1 / d}(d+2)((d+2) L(d ; r)+1)}<\frac{1}{2(d+2)(r-2)},
$$

and since $\sqrt{1+t}<1+t / 2$ for positive $t$, (15) implies

$$
z<c R+\frac{d-1}{2}+\frac{1}{2}\left(\frac{d-1}{2}\right)^{2}\left(\frac{1}{c}-c\right) \frac{1}{R} .
$$

By the bounds of $c$ and definition of $L(d ; r)$ we have further that

$$
\begin{aligned}
c R+\frac{d-1}{2} & <\left(\sqrt[d]{\frac{2}{d+2}}+\frac{1}{2(d+2)(r-2)}\right) R+\frac{d-1}{2} \\
& =L(d ; r)+\frac{R}{2(d+2)(r-2)} \\
& <L(d ; r)+\frac{1}{2(d+2)}
\end{aligned}
$$

and hence (16) implies

$$
z<L(d ; r)+\frac{1}{2(d+2)}+\frac{1}{2}\left(\frac{d-1}{2}\right)^{2}\left(\frac{1}{c}-c\right) \frac{1}{R} .
$$

Since $t \mapsto 1 / t-t$ is decreasing we have further

$$
\frac{1}{c}-c<\sqrt[d]{\frac{d+2}{2}}+\sqrt[d]{\frac{2}{d+2}}<\frac{2 \log (d+2)}{d}
$$

and so (17) implies the following.

Proposition 5.10 If $L(d ; r)$ is as defined in (14), then the real number solution $z$ to (11) satisfies

$$
0<z-L(d ; r)<\frac{1}{2(d+2)}+\frac{d \log (d+2)}{2(2 r-d-3)}
$$

for all natural numbers $d$ and $r$ with $d<r$.

Note: (i) By Proposition 5.10 we have for any $\varepsilon>0$ there is an integer $d_{\varepsilon}^{\prime}$ and a function $t \mapsto r_{\varepsilon}^{\prime}(t)$ such that for any $d \geq d_{\varepsilon}^{\prime}$ and $r \geq r_{\varepsilon}^{\prime}(d)$ then $0<z-L(d ; r)<\varepsilon$. (ii) Also, as $(1-\sqrt[d]{2 /(d+2)})(d-1) / 2=\log (d / 2) / 2+O\left(\log ^{2} d / d\right)$ we immediately obtain that the real number solution $z$ to (11) satisfies

$$
z=\sqrt[d]{\frac{2}{d+2}}(r-2)+\frac{\log (d / 2)}{2}+O\left(\frac{\log ^{2}(d)}{d}\right)+O\left(\frac{d \log (d)}{r}\right),
$$

for large $d$ and $r$ with $d<r$.

By the above Proposition 5.10 we now have the following result. 
Theorem 5.11 If a Minkowski sum $P_{\mathcal{F}}=\Delta_{F_{1}}+\Delta_{F_{2}}$ where $F_{1}, F_{2} \subseteq\{1, \ldots, r\}$ and $r_{1} \geq 1$ has the maximum discrepancy $\delta_{2 ;(0, d)}\left(P_{\mathcal{F}}\right)=\delta_{2 ;(0, d)}(r)$ between the number of $(0, d)$-chains of faces of $P_{\mathcal{F}}$ and the minimum number $\left(\begin{array}{c}r-1 \\ d\end{array}\right) f_{0}$ of $(0, d)$-chains in a simple polytope of the same dimension and with the same number $f_{0}$ of vertices, then we have $\left|r_{2}-r_{3}\right| \leq 1$ and

$$
\lceil L(d ; r)\rceil \leq r_{2}+r_{3} \leq\left\lfloor L(d ; r)+\frac{2 d+5}{2 d+4}+\frac{d \log (d+2)}{2(2 r-d-3)}\right\rfloor,
$$

where $L(d ; r)$ is as defined in (14).

Since the ring $\mathbb{Z}$ of the integers is a UFD, the proof of the following is an easy exercise.

Claim 5.12 For any integer $d \geq 2$ the number $\sqrt[d]{\frac{2}{d+2}}$ is irrational.

By the above Claim 5.12 and the Equidistribution Theorem by Hermann Weyl [7, p. 107], we have the following.

Observation 5.13 For every fixed integer $d \geq 2$ the set $\{L(d ; r) \bmod 1: r \in \mathbb{N}\}$ is uniformly distributed and hence a dense subset of the unit interval $[0,1]$ with respect to the usual topology of the reals.

Consider the upper bound of $r_{2}+r_{3}$ in Theorem 5.11. For every fixed $d \geq 1$ we see that if $r>d \log (d+2)$ then the real interval in which the integer $r_{2}+r_{3}$ is contained in is of length strictly less than 2 and hence there are at most two possibilities for the integer $r_{2}+r_{3}$. Observation 5.13 now implies there are infinitely many $r>d \log (d+2)$ for which the integer $r_{2}+r_{3}$ is uniquely determined by Theorem 5.11. In this case we know exactly when the maximum discrepancy occurs.

Corollary 5.14 With the setup from Theorem 5.11, if $\delta_{2 ;(0, d)}\left(P_{\mathcal{F}}\right)=\delta_{2 ;(0, d)}(r)$, then for all fixed $d \geq 1, r>d \log (d+2)$ and $i \in\{2,3\}$, we have

$$
\begin{aligned}
\left|r_{2}-r_{3}\right| & \leq 1 \\
\left|r_{2}+r_{3}-\lceil L(d ; r)\rceil\right| & \leq 1
\end{aligned}
$$

By symmetry we may assume $r_{2} \leq r_{3}$ to hold, in which case we have further there are infinitely many $r$ such that the maximum discrepancy $\delta_{2 ;(0, d)}(r)$ occurs exactly at

$$
\begin{aligned}
& r_{1}=r-\lfloor\lceil L(d ; r)\rceil / 2\rfloor-\lceil\lceil L(d ; r)\rceil / 2\rceil, \\
& r_{2}=\lfloor\lceil L(d ; r)\rceil / 2\rfloor, \\
& r_{3}=\lceil\lceil L(d ; r)\rceil / 2\rceil .
\end{aligned}
$$

REMARK: Although $r_{2}+r_{3}$ is for all $r>d \log (d+2)$ uniquely determined up to parity, we have by the uniform distribution property of $L(d ; r) \bmod 1$ in Observation 5.13 that for every fixed $d$, "most" $r>d \log (d+2)$ will determined $r_{1}, r_{2}$ and $r_{3}$ uniquely as stated in the latter display in Corollary 5.14. That is to say, the probability of picking $r$ that uniquely determines $r_{1}, r_{2}$ and $r_{3}$ can be made arbitrarily high by choosing large enough $d$ and large enough $r>d \log (d+2)$. 


\section{Acknowledgments}

The author would like to thank James F. Lawrence for helpful discussions regarding the theory of polytopes in general. Also, thanks to Bernd Sturmfels for introducing this investigation of Minkowski sum of standard simplices. Last but not least, sincere thanks to Walter Morris for his encouragement, interest and helpful discussions. Figure 1 is the same that appears in [8] and was drawn by Walter Morris.

\section{References}

[1] Ernst Steinitz, Über die Eulerschen Polyederreliationen, Archiv für Mathematik und Physik 11, $86-88,(1906)$.

[2] Margaret M. Bayer, Louis J. Billera, Generalized Dehn-Sommerville relations for polytopes, spheres and Eulerian partially ordered sets. Invent. Math. 79, no. 1, $143-157$, (1985).

[3] Margaret M. Bayer, Andrew Klapper, A new index for polytopes, Discrete Comput. Geom. 6, no. $1,33-47,(1991)$.

[4] Richard Ehrenborg, Margaret Readdy, Coproducts and the cd-index. J. Algebraic Combin. 8, no. 3, $273-299$, (1998).

[5] Richard Ehrenborg, Harold Fox, Inequalities for $c d$-indices of joins and products of polytopes. Combinatorica, 23, no 3, 427-452, (2003).

[6] Gil Kalai, The number of faces of centrally-symmetric polytopes. Graphs Combin., 5, no. 1, $389-391$, (1989).

[7] Elias M. Stein, Rami Shakarchi, Fourier Analysis, An Introduction. Princeton Lectures in Analysis I, Princeton University Press, Princeton, New Jersey, (2003).

[8] Geir Agnarsson and Walter Morris, On Minkowski sum of simplices, Annals of Combinatorics, 13, $271-287,(2009)$.

[9] E. Gawrilow and M. Joswig, Polymake: a framework for analyzing convex polytopes, in Polytopes - Combinatorics and Computation, eds. G. Kalai and G. M. Ziegler, Birkhaüser, pp. $43-74,(2000)$.

[10] Günter M. Ziegler, Lectures on polytopes, Graduate Texts in Mathematics, GTM - 152, Springer-Verlag Inc., New York, (1995).

September 21, 2018 